\title{
Estimation of Dynamic Discrete Models from Time Aggregated Data *
}

\author{
Han Hong ${ }^{a}$ \\ Weiming $\mathrm{Li}^{b}$ \\ First draft: September 2011 \\ Current draft: November 2014
}

Boyu Wang ${ }^{c}$

\begin{abstract}
An important component in dynamic discrete choice models and dynamic discrete games is the transition density of state variables from the current period to the next period. Most empirical dynamic discrete choice models identify the theoretical time interval in the behavioral model with that observed in the data set. However, many empirical data sets are time aggregated. In this paper, we show that when the time interval in the behavioral theory model differs from that in the observed data, difficulties with nonparametric identification and specification arise. In addition, we study the properties of parametric maximum likelihood estimators and flexible semiparametric estimators of the transition density in dynamic discrete models with time aggregated data sets.
\end{abstract}

Keywords: Dynamic Discrete Choice Models, Maximum Likelihood Estimator, Semiparametric Methods.

JEL Classification: E00, G12, C51, C52

${ }^{*}$ We acknowledge generous support by the National Science Foundation (SES 1024504, 1164589) and SIEPR. We also thank the associate editor and two anonymous referees for helpful comments.

${ }^{a}$ Department of Economics Stanford University, Stanford, CA 94305, USA.

${ }^{b}$ Capital University of Economics and Business, Beijing, China.

${ }^{c}$ RadiumOne Inc. 55 Second Street, San Francisco, CA 94105, USA. 


\section{Introduction}

In dynamic discrete choice models and discrete games, developed in Rust (1987) and Hotz and Miller (1993), agents solve an optimal dynamic action as a function of the current state variables. The state variables evolve according to a law of motion that also depends on the actions by the agents. A recent literature generalizes to dynamic games and develops nonparametric identification results and flexible semiparametric estimators, including contributions from, among others, Bajari, Benkard, and Levin (2007), Aguirregabiria and Mira (2007), Berry, Pakes, and Ostrovsky (2003), Pesendorfer and Schmidt-Dengler (2010), Jenkins, Liu, McFadden, and Matzkin (2004), Magnac and Thesmar (2002), Kasahara and Shimotsu (2008), Hu and Shum (2012), Arcidiacono and Miller (2011), Norets (2009), Imai, Jain, and Ching (2009) and Bajari, Chernozhukov, Hong, and Nekipelov (2009).

A potential important issue in empirically estimating dynamic discrete choice models is that there is often a discrepancy between the frequency of observations in the data set and the decision frequency in the behavior model. Often times individual behaviors are reported in prespecified time intervals in each data set. This can be daily, monthly, quarterly or annually, etc. For example, the Health and Retirement Survey was conducted once every two years. This requires that a researcher adapts the frequency of the decision making in the dynamic discrete choice model to the observed time intervals reported in the data set (Fang and Wang (2010)). Such an approach will lead to inconsistent estimates of the utility parameters when the decision time model is misspecified. Other applications of dynamic discrete models include Rust (1987), Jenkins, Liu, McFadden, and Matzkin (2004) and Ryan (2012).

Exceptions are Doraszelski and Judd (2012) and Arcidiacono, Bayer, Blevins, and Ellickson (2012), who formulate and estimates continuous time dynamic discrete models. In this paper we consider equal-spaced discrete time models, in which the data frequency is coarser than the model frequency. Our results complement those in Blevins (2013) and Blevins (2014), who provides identification results for continuous time models using discretely observed data using the logarithmic root of the discrete time state transition matrix.

The structural parameters in a dynamic discrete choice model include the discount rate, the error distribution, the period utility function and the state variable transition density. 
When the discount rate and the error distribution are known as is conventionally assumed in the literature, the dynamic discrete model is exactly identified. In particular, the state variable transition density is directly identified and can be estimated from the data. The period utility function can then be recovered from the reduced form choice probabilities under a set of exclusion restrictions.

These results no longer hold when only time aggregated data is available where the data frequency differs from the model frequency. With time aggregated data, only multiperiod state variable transition density can be directly identified and recovered from the data set. Multi-period transition density, however, is a convoluted function of the singleperiod transition density and the conditional choice probabilities of actions as a function of the state variables. The inverse mapping of this relation might not exist or might admit multiple solutions. As a consequence, the single period state transition density can be either correctly specified and identified, or misspecified, or unidentified. Even when the population model is correctly specified, nonparametric methods of identification and estimation that are applicable for disaggregated time data still can not be applied directly to aggregate time data sets.

Parametric and semiparametric maximum likelihood methods can be applied to aggregated time data when the population model is correctly specified and identified. We discuss the adaptation of the estimation methods for dynamic discrete models developed in Rust (1987) and Hotz and Miller (1993) to time aggregate data sets, and present their statistical and computational properties. The EM algorithm (Arcidiacono and Jones (2003) and Arcidiacono and Miller (2011)) can be utilized to efficiently compute the MLE estimators. We find that an estimator based on aggregated time data might achieve more statistical efficiency than an estimator based on regularly spaced observations. Numerical simulations show that the performance of likelihood based estimators in finite samples accords with the predictions of the asymptotic theory.

\section{Model and Data}

We consider a stationary dynamic environment. Let $t=0,1, \ldots, \infty$ denote time. The notations to be introduced below will apply to both single agent dynamic discrete choice settings 
and multi-agent dynamic discrete games with $n$ agents, in which case the decision making of forward looking rational agents is assumed to be consistent with a dynamic program as in Rust (1987) or a Markov perfect dynamic equilibrium.

In each time period $t$, agent $j$ chooses an action $a_{j t}$ from $\mathcal{A}=\{1, \cdots L\}$. Its observed state variable is denoted by $x_{j t}$, taking value from a finite set of $\mathcal{X}=\{0,1 \cdots K\}$. Further, let $a_{t}=\left(a_{j t}, \forall j\right) \in \mathcal{A}^{m}$ and $x_{t}=\left(x_{j t}, \forall j\right) \in \mathcal{X}^{m}$ be the action and state profile vectors of all agents. Also let $a_{-j}$ be a vector of strategies for all players excluding $j$, and similarly for $x_{-j}$. While $x_{j t}$ can be perfectly observed by both the agents and the econometrician subject to the data periodicity to be discussed below, there is a set of unobserved state variable $\epsilon_{j t}\left(a_{j t}\right)$ observable only by agent $j$ as private information. Throughout the paper we maintain the conventional conditional independence and distribution assumptions following Rust (1987).

ASSUMPTION 1 The error terms $\epsilon_{j t}\left(a_{j t}\right)$ are identically and independently distributed across $t, j$ and $a_{j t}$ and follow Type I extreme value distribution.

ASSUMPTION 2 Conditional on $x_{t}$ and $a_{t}, x_{t+1}$ is independent of $\epsilon_{t}$.

The complete state variable of agent $j$ at time $t$ is $s_{j t}=\left(x_{j t}, \epsilon_{j t}\right)$. In each period agent $j$ derives utility $u_{j}\left(a_{t}, x_{t}, \epsilon_{j t}\right)=\Pi_{j}\left(a_{j t}, a_{-j t}, x_{t} ; \theta_{u}\right)+\epsilon_{j t}\left(a_{j t}\right)$. The period mean utility function $\Pi_{j}\left(a_{j t}, a_{-j t}, x_{t} ; \theta_{u}\right)$ is described by a parameter vector $\theta_{u}$, which includes the nonparametric specification as a special case when the state variables are discrete. Agents are forward looking with a given discount rate $\beta$. In equilibrium, they choose best response $a_{j t}$ for a given $x_{t}$ and $\epsilon_{j t}$ to maximize a discounted sum of expected future utility functions,

$$
E\left[\sum_{\tau=0}^{\infty} \beta^{\tau} u_{j}\left(a_{t+\tau}, x_{t+\tau}, \epsilon_{t+\tau}\right) \mid a_{j t}, x_{t}, \epsilon_{j t}\right] .
$$

The Markov perfect equilibrium conditional choice probabilities $\sigma_{j}\left(a_{j} \mid x\right)$ are obtained by integrating out $\epsilon_{j t}$ in the optimal response function $a_{j t}\left(x_{t}, \epsilon_{j t}\right)$.

Because of the private information assumption, the dynamic discrete game is observationally equivalent to a single agent dynamic discrete model, in which the period expected utility function of agent $j$ is given by $\Pi_{j}\left(a_{j}, x\right)=\sum_{a_{-j} \in A_{-j}} \Pi_{j}\left(a_{j}, a_{-j}, x\right) \sigma_{-j}\left(a_{-j} \mid x\right)$, and in which agent $j$ follows the Bellman principle of stochastic dynamic programming

$$
W_{j}\left(x_{t}, \epsilon_{j t} ; \sigma_{-j}\right)=\max _{a_{j t} \in \mathcal{A}}\left\{\Pi_{j}\left(a_{j t}, x_{t}\right)+\epsilon_{j t}+\beta \int_{x^{\prime}, \epsilon^{\prime}} W_{j}\left(x^{\prime}, \epsilon^{\prime} ; \sigma_{-j}\right) d F\left(x^{\prime}, \epsilon^{\prime} \mid a_{j t}, x_{t}, \epsilon_{j t}\right)\right\}
$$


where the value function for player $j, W_{j}\left(\cdot, \cdot ; \sigma_{-j}\right)$, is defined as the fixed point of the above functional equation.

Because of assumptions 1 and 2, the Bellman principle can be expressed through a choice specific value function $V_{j}\left(a_{j}, x\right)$ defined as

$$
V_{j}\left(a_{j}, x\right)=\Pi_{j}\left(a_{j}, x ; \theta_{u}\right)+\beta \int_{x^{\prime}} V_{j}\left(x^{\prime}\right) d F\left(x^{\prime} \mid a_{j}, x\right)
$$

where the social surplus function or ex ante value function is defined by

$$
V_{j}(x)=\int W_{j}\left(x, \epsilon_{j} ; \sigma\right) f\left(\epsilon_{j}\right) d \epsilon_{j}
$$

Under assumption 1, the social surplus function is in turn related to the choice specific value functions through the following relation

$$
V_{j}(x)=G\left(V_{j}\left(a_{j}, x\right), \forall a_{j}\right) \equiv \log \sum_{a_{i}=0}^{L} \exp \left(V_{j}\left(a_{j}, x\right)\right) .
$$

In a Markov perfect equilibrium of a discrete game, the transition distribution of the observed state variables satisfies, by conditional independence

$$
F\left(x_{t+1} \mid a_{j t}, x_{t}, \epsilon_{j t}\right)=F\left(x_{t+1} \mid a_{j t}, x_{t}\right)=\sum_{a_{-j t}} g\left(x_{t+1} \mid x_{t}, a_{j t}, a_{-j t}\right) \cdot \sigma_{-j}\left(a_{-j t} \mid x_{t}\right)
$$

where $g\left(x_{t+1} \mid x_{t}, a_{j t}, a_{-j t}\right)$ denotes the single period conditional density of the next period state variables given the current state variables and actions. In the following, we will focus on a single agent dynamic model with infinite horizon and stationarity in which $j=1, \ldots, n$ denotes individuals operating in $n$ independent markets.

Estimation of a dynamic discrete choice model requires a cross section of observations on the choice profile and state variables $\left(a_{t}, x_{t}\right)$, which provides information about the conditional choice probabilities $p\left(a_{t} \mid x_{t}\right)$, and data that can be used to recover the transition density of the observed state variables $F\left(x_{t+1} \mid a_{i t}, x_{t}, \epsilon_{i t}\right)$. In a typical stationary model in which the frequency of data availability coincides with the model decision frequency, this is given by the conditional distribution of $x_{t+1}$ given $a_{t}$ and $x_{t}$. We denote a balanced panel data of non time aggregated data set by

$$
D=\left\{\left(a_{i, 0}, x_{i, 0}\right),\left(a_{i, 1}, x_{i, 1}\right), \cdots,\left(a_{i, T}, x_{i, T}\right)\right\}_{1 \leq i \leq n}
$$


Instead of this conventional setting, in the rest of the paper we focus on time aggregated data in which the data frequency is coarser than the model frequency. In the model, time increments by each period. However, data is only available in every $r$ periods. In other words, for $r \geq 1$, we assume availability of only a balanced time aggregated panel of observations

$$
D_{r}=\left\{\left(a_{i, 0}, x_{i, 0}\right),\left(a_{i, r}, x_{i, r}\right), \cdots,\left(a_{i, M r}, x_{i, M r}\right)\right\}_{1 \leq i \leq n}
$$

where $M=\lfloor T / r\rfloor$. The focus of the remaining investigation is on the identification and estimation of the transition density $F\left(x_{t+1} \mid a_{t}, x_{t}\right)$, from time aggregated data sets $D_{r}$, which is required for the estimation of the structural parameters in a subsequent stage. We will call this case the reduced model.

\section{Nonparametric Identification of the Reduced Model}

In the reduced model, the state variables $x \equiv x_{t}, x^{(r)} \equiv x_{t+r}$ at time $t$ and $t+r$, as well as the action $a_{t}$ at time $t$ are assumed to be fully observed for the purpose of discussing nonparametric identification. The transition matrix of state variables at time period $r$ given current action $a$ is denoted $F_{a}^{(r)}$ and is defined by $\left[F_{a}^{(r)}\right]_{i j} \equiv f\left(x^{(r)}=j \mid x=i, a\right)$. This can be directly estimated from the data. However, to recover the structural model we need to identify the single period conditional transition matrix, denoted as $F_{a} \equiv F_{a}^{(1)}$. Also denote $x^{\prime} \equiv x^{(1)}=x_{t+1}$. Since $x^{\prime}$ is unknown to us, we cannot estimate $F_{a}$ directly from the data.

For further discussion we need to define additional notations. The $K \times K$ diagonal matrix of conditional action probabilities $\Lambda_{a}$ is defined as: $\Lambda_{a} \equiv \operatorname{diag}\{\sigma(a \mid x=i)\}$. Here $\sigma(a \mid x=i)$ is probability for the agent to choose $a$ conditional on state $x=i . \Lambda_{a}$ can be estimated directly from the data, with zero off diagonal elements. Next, we define the $r$ period transition matrix, unconditional on the actions taken, as $\left[T^{(r)}\right]_{i j}=f\left(x^{(r)}=j \mid x=i\right)$. Note that

$$
T^{(r)} \equiv\left(\sum_{l=1}^{L} \Lambda_{l} F_{l}^{(r)}\right)
$$

This is because for each $i, j$ element,

$$
\left(\sum_{l=1}^{L} \Lambda_{l} F_{l}^{(r)}\right)_{i j}=\sum_{l=1}^{L}\left(\Lambda_{l} F_{l}^{(r)}\right)_{i j}=\sum_{l=1}^{L}\left(\Lambda_{l}\right)_{i i}\left(F_{l}^{(r)}\right)_{i j}
$$




$$
=\sum_{l=1}^{L} \sigma(a=l \mid x=i) \cdot f\left(x^{(r)}=j \mid x=i, a=l\right)=f\left(x^{(r)}=j \mid x=i\right)=\left[T^{(r)}\right]_{i j} .
$$

We seek to identify $F_{a}$ from the knowledge of $F_{a}^{(r)}, T^{(r)}$ and $\Lambda_{a}$, each of which can be estimated from the data.

LEMMA 1 The transition matrix $F_{a}^{(r)}$ can be represented as follows:

$$
F_{a}^{(r)}=F_{a} \sum_{l=1}^{L} \Lambda_{l} F_{l}^{(r-1)}=F_{a} T^{(r-1)} .
$$

The first equality in lemma 1 follows from considering each $i, j$ element. The left hand side is $f\left(s^{(r)}=j \mid s=i, a=a\right)$ and the right hand side is

$$
\sum_{l=1}^{L} \sum_{k=1}^{K} f\left(x^{\prime}=k \mid x=i, a=a\right) \sigma(a=l \mid x=k) f\left(x^{(r)}=j \mid x^{\prime}=k, a=l\right) .
$$

The first equality then follows from the definition of the Markov process. The second equality follows from equation (4).

Noting that $T^{(r)}=T^{(1)} T^{(r-1)}$ and $T^{(r)}=\left[T^{(1)}\right]^{r}$, we obtain the following relation.

Theorem 3.1 The transition matrix $F_{a}^{(r)}$ can be represented by $F_{a}$ and $\Lambda_{a}$ as follows:

$$
F_{a}^{(r)}=F_{a}\left(\sum_{l=1}^{L} \Lambda_{l} F_{l}\right)^{r-1} \equiv F_{a}\left(T^{(1)}\right)^{r-1}=F_{a} T^{(r-1)}, \quad \forall a \in \mathcal{A}
$$

Proof: We prove by mathematical induction. First, it is obviously correct from Lemma 1 if $r=2: F_{a}^{(2)}=F_{a} \sum_{l=1}^{L} \Lambda_{l} F_{l}$. Assume Theorem 3.1 is correct for $r$, Thus, for $r+1$, we use Lemma 1 and the inductive hypothesis at $r$,

$$
F_{a}^{(r+1)}=F_{a} \sum_{l=1}^{L} \Lambda_{l} F_{l}^{(r)}=F_{a} \sum_{l=1}^{L} \Lambda_{l}\left[F_{l}\left(\sum_{j=1}^{L} \Lambda_{j} F_{j}\right)^{r-1}\right]=F_{a}\left(\sum_{l=1}^{L} \Lambda_{l} F_{l}\right)^{r}
$$

As a result, Theorem 3.1 is correct for any $r$.

From the data, we can recover $F_{l}^{(r)}$ and $\Lambda_{l}$, and want to estimate $F_{a}$. Theorem 3.1 provides the following explicit solution.

Theorem 3.2 The transition matrix $F_{a}$ can be recovered from $F_{a}^{(r)}$ and $\Lambda_{a}$ as follows:

$$
F_{a}=F_{a}^{(r)}\left(\sum_{l=1}^{L} \Lambda_{l} F_{l}^{(r)}\right)^{-\frac{r-1}{r}}=F_{a}^{(r)}\left(T^{(r)}\right)^{-\frac{r-1}{r}}
$$

whenever the solution the matrix inversion and root operation above exists and is unique for each $a \in \mathcal{A}$. 
Proof: It follows from Theorem 3.1 that: $F_{b}^{-1} F_{b}^{(r)}=F_{a}^{-1} F_{a}^{(r)}=T^{(r-1)}, \quad \forall a, b \in \mathcal{A}$. So any $F_{b}$ can be represented by $F_{a}$ as:

$$
F_{b}=F_{b}^{(r)}\left[F_{a}^{(r)}\right]^{-1} F_{a}, \quad \forall a, b \in \mathcal{A}
$$

Substitute equation (7) into Theorem 3.1:

$$
F_{a}^{(r)}=F_{a}\left(\sum_{l=1}^{L} \Lambda_{l} F_{l}^{(r)}\left[F_{a}^{(r)}\right]^{-1} F_{a}\right)^{r-1}
$$

Pre-multiply both sides of Equation (8) by $\sum_{l=1}^{L} \Lambda_{l} F_{l}^{(r)}\left[F_{a}^{(r)}\right]^{-1}$ :

$$
\sum_{l=1}^{L} \Lambda_{l} F_{l}^{(r)}=\left(\sum_{l=1}^{L} \Lambda_{l} F_{l}^{(r)}\left[F_{a}^{(r)}\right]^{-1} F_{a}\right)^{r}
$$

As a result, $F_{a}$ is solved as:

$$
F_{a}=F_{a}^{(r)}\left(\sum_{l=1}^{L} \Lambda_{l} F_{l}^{(r)}\right)^{-1}\left(\sum_{l=1}^{L} \Lambda_{l} F_{l}^{(r)}\right)^{\frac{1}{r}}=F_{a}^{(r)}\left(\sum_{l=1}^{L} \Lambda_{l} F_{l}^{(r)}\right)^{-1+\frac{1}{r}}
$$

Theorem 3.2 suggests the possibility of recovering $F_{a}$. Both $F_{a}^{(r)}$ and $T^{(r)}$ are stochastic matrices that can be estimated directly from the data nonparametrically. Implementing 3.2 requires inverting the $r$ th root of a stochastic matrix, which however poses at least two types of difficulty. The first issue is existence. There may not exist a stochastic matrix solution to Theorem 3.2. The second issue is nonuniquess of the $r$ th root of a matrix, inducing multiple solutions to Theorem 3.2 .

Theorem 3.3 When a model is correctly specified, there must be at least one stochastic matrix that satisfies equation (6) at the true value of $F_{a}^{(r)}$ and $T^{(r)}$. A correctly specified model does not have to be identified. A model is not identified when there are multiple solutions to equation (6).

While theorem 3.3 is a population construct, in finite sample failing to find a stochastic root of equation (6) can indicate either the sampling uncertainty in the estimates of $\hat{F}_{a}^{(r)}$ and $\hat{T}^{(r)}$, or the possibility of model misspecification, which also suggests the basis of a model specification test. Even if the model is correctly specified, the model is not identified without further assumptions. 
To illustrate the complexity of the uniqueness and existence of the $r$ th root of a stochastic matrix, consider a simple example of finding the $r$ th root of a $2 \times 2$ stochastic matrix: $X^{r}=A$. The problem is equivalent to solving $x, y \in[0,1]$ from $a, b \in[0,1]$ :

$$
X^{r}=\left[\begin{array}{cc}
x & 1-x \\
1-y & y
\end{array}\right]^{r}=\left[\begin{array}{cc}
a & 1-a \\
1-b & b
\end{array}\right]=A
$$

An explicit solution is showed in He and Gunn (2003). We provide an alternative derivation in the following proposition.

Proposition 3.1 Except when $a=b=1$, the solution of Equation 10 is as follows:

1. There is no stochastic matrix solution either when $r$ is odd, $a+b+\min \left\{\left(\frac{1-a}{1-b}\right)^{r},\left(\frac{1-b}{1-a}\right)^{r}\right\}<$ 1 ; or when $r$ is even, $a+b<1$.

2. There is a unique stochastic matrix solution either when $r$ is odd,

$$
a+b+\min \left\{\left(\frac{1-a}{1-b}\right)^{r},\left(\frac{1-b}{1-a}\right)^{r}\right\} \geq 1
$$

or when $r$ is even, $a+b>1+\min \left\{\left(\frac{1-a}{1-b}\right)^{r},\left(\frac{1-b}{1-a}\right)^{r}\right\}$; or when $r$ is even, $a+b=1$, given by

$$
X=\left[\begin{array}{ll}
\frac{(1-b)+(1-a) \sqrt[r]{a+b-1}}{(1-a)+(1-b)} & \frac{(1-a)-(1-a) \sqrt[r]{a+b-1}}{(1-a)+(1-b)} \\
\frac{(1-b)-(1-b) \sqrt[r]{a+b-1}}{(1-a)+(1-b)} & \frac{(1-a)+(1-b) \sqrt[r]{a+b-1}}{(1-a)+(1-b)}
\end{array}\right]
$$

3. There are two stochastic matrix solutions $X$ when $r$ is even, $1<a+b \leq 1+$ $\min \left\{\left(\frac{1-a}{1-b}\right)^{r},\left(\frac{1-b}{1-a}\right)^{r}\right\}$, given by

$$
\left[\begin{array}{cc}
\frac{(1-b)+(1-a) \sqrt[r]{a+b-1}}{(1-a)+(1-b)} & \frac{(1-a)-(1-a) \sqrt[r]{a+b-1}}{(1-a)+(1-b)} \\
\frac{(1-b)-(1-b) \sqrt[r]{a+b-1}}{(1-a)+(1-b)} & \frac{(1-a)+(1-b) \sqrt[r]{a+b-1}}{(1-a)+(1-b)}
\end{array}\right] \quad \text { and } \quad\left[\begin{array}{cc}
\frac{(1-b)-(1-a) \sqrt[r]{a+b-1}}{(1-a)+(1-b)} & \frac{(1-a)+(1-a) \sqrt[r]{a+b-1}}{(1-a)+(1-b)} \\
\frac{(1-b)+(1-b) \sqrt[r]{a+b-1}}{(1-a)+(1-b)} & \frac{(1-a)-(1-b) \sqrt[r]{a+b-1}}{(1-a)+(1-b)}
\end{array}\right]
$$

The three regions of parameters $(a, b)$ in Proposition 3.1 corresponding to no solution, unique solution, and multiple solution are illustrated in figure 1.

Figure 1 about here.

A few remarks are in order. First, in the special case of $A=I$, there is always a solution $X=I$ for all $r$, which is also unique when $r$ is odd. However, when $r$ is even, there is also a second solution which permutes the identity matrix,

$$
X=\left[\begin{array}{ll}
0 & 1 \\
1 & 0
\end{array}\right]
$$


On the other hand, when either $a=1, b \in(0,1)$, or $a \in(0,1), b=1$, there is a unique stochastic solution for all $r$. We also note that the previous solution concept is confined to stochastic matrices. There may also exist non-stochastic matrix roots, which can contain negative or complex elements.

Only case 3 in proposition 3.1 admits multiple stochastic roots. In this case, note that one of the two solutions has a dominant diagonal. This suggests the possibility of selecting solutions based on classifying their properties. In particular, Lin (2011) classifies matrix roots into primary and nonprimary roots. A primary $p$ th root of a matrix $A$ is the $p$ th root that is expressible as polynomials in $A$. A matrix $A$ with no nonpositive eigenvalues has a unique $p$ th root $X$ with its spectrum in the wedge $-\pi / p<\arg (\lambda(X))<\pi / p$, called the principal $p$ th root. While each stochastic matrix has no nonpositive eigenvalues and only has one unique principal $p$ th root, its principal $p$ th root may not be stochastic, in which case other primary or nonprimary roots might be stochastic.

The difficulty with existence and multiplicity of matrix roots suggests that a nonparametric estimation approach based on inverting the $r$ th period transition matrix is difficult or infeasible. Even when the state variables are finitely discrete, to incorporate the model restriction that the solution to equation (6) in Theorem 3.2 is a stochastic matrix, it is necessary to rely on parametric estimation procedures. In the rest of the paper we focus on parametric and maximum likelihood based estimation approaches.

Recently, another concept of the root of a matrix $A$ called eventual positivity was investigated by McDonald, P., and Tsatsomeros (2013). A matrix $A$ is eventually positive if there exists a natural number $p$ such that $A^{k}$ is entry-wise positive for all $k \geq p$. The eventual stochasticity property is further defined if $A$ and $A^{k}$ are stochastic matrices. The main applicable result of eventual stochasticity is the number of eventually stochastic primary $r$-th roots of a nonsingular stochastic matrix $A$. Assume $A$ has $n_{1}$ distinct positive real eigenvalues, $n_{2}$ distinct negative real eigenvalues and $n_{c}$ distinct complex-conjugate pairs of eigenvalues. If $r$ is even, then there are $2^{n_{1}-1} r^{n_{c}}$ such roots when $n_{2}=0$ and no such roots when $n_{2}>0$; if $r$ is odd, there are always $r^{n_{c}}$ such roots. Some other related results are also explained in McDonald, P., and Tsatsomeros (2013).

A straightforward characterization of the root calculation is available when the uncon- 
ditional $r$ th period state transition matrix admits a diagonal representation, in that it can be written as $T^{(r)}=P^{-1} \Lambda P$, where $P$ is an orthonormal matrix of eigenvectors and $\Lambda$ is a diagonal matrix of eigenvalues. In this case $T=P^{-1} \Lambda^{\frac{1}{r}} P$ where $\Lambda^{\frac{1}{r}}$ is a diagonal matrix of the $r$ th roots of $\Lambda$. Multiple roots of $\Lambda$ generates multiple roots of $T^{(r)}$.

In addition to the issues discussed above, the results in this section for an infinite horizon stationary model do not apply in models with finite horizons. In finite horizon models typically both the conditional choice probability matrix $\Lambda_{a}$ and the state transition probability matrix $F_{a}$ are time varying. They require complete observations of panel data for identification. Even when the state transition probability matrix $F_{a}$ is assumed to be time-invariant in a finite horizon model, the conditional choice probability matrix $\Lambda_{a}$ is still time varying and mostly unobserved, which invalidates the previous identification arguments.

\subsection{A Note on Maximum Likelihood Estimation}

Before we discuss (pseudo) maximum likelihood estimation of the structural model, we comment on the relation between maximum likelihood estimation and nonparametric methods in the presence of time aggregation. When single period observed transition data is available and when the state variables are finitely discrete, nonparametric maximum likelihood estimates of the elements of the transition matrix are given by empirical frequency counts. This equivalence does not hold however when only time aggregated observations are available.

To illustrate, consider estimating the single-period and $r$ period unconditional (on actions) transition matrix $[T]_{i j}$ and $\left[T^{(r)}\right]_{i j}$ from $r$ period reduced data. For a given set of $r$ period transition data, For a given Denote $\left[N^{(r)}\right]$ the empirical frequency matrix with elements $n_{i j}^{r}$, the number of observations with $x_{t}=i$ and $x_{t+r}=j$. It is standard that the elements of the maximum likelihood estimate of the state transition probability matrix $\left[T^{(r)}\right]_{i j}$ (without accounting for the dependence on parameters in $[T]_{i j}$ ), as defined by,

$$
\left[\widehat{T^{(r)}}\right]_{i j}=\arg \max _{p_{i j}} \sum_{i=1}^{K} \sum_{j=1}^{K} n_{i j}^{r} \log \left(p_{i j}\right) \text { such that } \sum_{j=1}^{K} p_{i j}=1 \quad \text { for each } i \text { and } p_{i j} \geq 0
$$

are given by the empirical frequency counts: $\left[\hat{T}^{(r)}\right]_{i j}=\frac{n_{i j}^{r}}{\sum_{k=1}^{K} n_{i k}^{r}}$. 
The maximum likelihood estimate of $[T]_{i j}$, given by

$$
\hat{T}=\arg \max _{A} \sum_{i=1}^{K} \sum_{j=1}^{K} n_{i j}^{r} \log \left(\left[A^{r}\right]_{i j}\right)
$$

such that $A_{i j} \geq 0$ and $\sum_{j=1}^{K} A_{i j}=1$ for each $i$, however does not necessarily satisfy

$$
\left[\hat{T}^{r}\right]_{i j}=\left[\widehat{T^{(r)}}\right]_{i j}
$$

In other words, the $r$ period transition matrix implied by the single period maximum likelihood estimate $\hat{T}^{r}$ is not necessarily the empirical frequency estimates of $\left[\widehat{T^{(r)}}\right]_{i j}$. A unique maximum likelihood estimate of $\hat{T}$ exists and satisfies the relation in equation (12) only when $\left[\widehat{T^{(r)}}\right]_{i j}$ has a unique stochastic root. When $\left[\widehat{T^{(r)}}\right]_{i j}$ has multiple stochastic roots, the likelihood function in $\hat{T}^{r}$ is also multi-modal, with each candidate solution of $\hat{T}^{r}$ satisfying equation (12). Finally, equation (12) does not hold when $\left[\widehat{\left.T^{(r)}\right)}\right]_{i j}$ has no stochastic root.

In the diagonally dominant case, $\hat{T}$ can be estimated by taking its unique $r$-th principal root. The consistency of this approach can be justified by the classical continuous mapping theorem by showing that taking the $r$-th principal root of a matrix is a continuous operation in a matrix norm. We consider the following matrix $\|\cdot\|_{\infty}$ norm

$$
\|A\|_{\infty}=\max _{1 \leq i \leq m} \sum_{j=1}^{n}\left|a_{i j}\right| .
$$

The matrix directional derivative $L_{1 / r}(T, E)$ (also known as Frechet derivative) with regard to the $r$ th principal root matrix function in the direction $E$ ( $E$ is of the same size as $T$ ) is defined as a linear operator such that

$$
(T+E)^{1 / r}-T^{1 / r}-L_{1 / r}(T, E)=o\left(\|E\|_{\infty}\right) .
$$

The following lemma due to Cardoso (2012) corroborates the above argument.

LEMMA 2 For a strictly diagonal dominant stochastic matrix $T$ and its principal root $T^{1 / r}$ and for $q \geq\left(2\|T\|_{\infty}\right)^{1 / r}$, the following holds,

$$
\left\|L_{1 / r}(T, E)\right\|_{\infty} \leq C_{r}\|E\|_{\infty}
$$


where $C_{r}$ is given as:

$$
C_{r}=\frac{r \sin (\pi / r)}{\pi}\left(\int_{[0, q]} t^{r}\left\|\left(t^{r} I+T\right)^{-1}\right\|_{\infty}^{2} d t+\frac{2 q^{1-r}}{r-1}\right) .
$$

Please note that the other condition mentioned in Cardoso (2012), $\|T-I\|_{\infty}<1$, holds for any strictly diagonally dominant stochastic matrix $T$. It follows that the induced operator norm $\left\|L_{1 / r}(T)\right\|_{\infty}$ can be bounded by $C_{r}$ which only depends on $r$. Furthermore, the upper bound can be decomposed into two parts based on some truncation point $q \geq 2\left(\|T\|_{\infty}\right)^{1 / r}$ : the first part in the integral form and the second part a term with exponential decay rate.

Statistically, the above inequality claims that when MLE is employed to estimate T, there is inevitably a discrepancy $E$ between $\hat{T}$ and $T$, but when the $r$-th principal root function is applied to $\hat{T}$, the discrepancy between $(\hat{T})^{1 / r}$ and $T^{1 / r}$ is controlled by $E$. And since by MLE's consistency property, $\|E\|_{\infty} \rightarrow 0$ when the sample size goes to infinity, the consistency of $(\hat{T})^{1 / r}$ follows as long as $T$ is strictly diagonally dominant.

\section{Structural Estimation}

Following the literature, two common approaches to estimating dynamic discrete choice models are the nested fixed point estimator of Rust (1987) and the multistep parametric estimator pioneered by Hotz and Miller (1993) and extensively explored by the recent literature. Both of these methods can be adapted to account for time aggregated observations of the form described at the end of section 2.

\subsection{Nested Fixed Point Maximum Likelihood Estimator}

With an aggregated data set $D_{r}$, the loglikelihood function for the nested fixed approach takes the form of

$$
\log \ell\left(\theta \mid D_{r}\right)=\underbrace{\sum_{i=1}^{n} \sum_{\tau=1}^{M} \log \sigma\left(a_{i, \tau r} \mid x_{i, \tau r} ; \theta\right)}_{\ell^{C C P}\left(\theta \mid D_{r}\right)}+\underbrace{\sum_{i=1}^{n} \sum_{\tau=0}^{M-1} \log f\left(x_{i,(\tau+1) r} \mid x_{i, \tau r}, a_{i, \tau r} ; \theta\right)}_{\ell^{M T P}\left(\theta \mid D_{r}\right)} .
$$

In the above, $\ell^{C C P}\left(\theta \mid D_{r}\right)$ is the likelihood contribution from the conditional action choice probabilities; $\ell^{M T P}\left(\theta \mid D_{r}\right)$ is the likelihood contribution from the $r$-period aggregated Markov 
transition probabilities $f(\cdot \mid \cdot, \cdot ; \theta)$. Unlike the likelihood function with complete data $D$ which is not time aggregated and where the second component only depends on $\theta_{g}$, the parameters in the transition probability matrix,

$$
\log \ell(\theta \mid D)=\underbrace{\sum_{i=1}^{n} \sum_{t=0}^{T} \log \sigma\left(a_{i t} \mid x_{i t} ; \theta\right)}_{\ell^{C C P}\left(\theta_{u}, \theta_{g} \mid D\right)}+\underbrace{\sum_{i=1}^{n} \sum_{t=0}^{T-1} \log g\left(x_{i, t+1} \mid x_{i, t}, a_{i, t} ; \theta_{g}\right)}_{\ell^{M T P}\left(\theta_{g} \mid D_{r}\right)},
$$

the parameters in the one period transition density, both $\ell^{C C P}\left(\theta \mid D_{r}\right)$ and $\ell^{M T P}\left(\theta \mid D_{r}\right)$ depend on both the utility and transition parameters in $\theta$. In the above,

$$
\sigma\left(a_{i, \tau r} \mid x_{i, \tau r} ; \theta\right)=\frac{\exp \left[V_{i}\left(a_{i}, x_{i, \tau r} ; \theta\right)\right]}{\sum_{l=1}^{L} \exp \left[V_{i}\left(l, x_{i, \tau r} ; \theta\right)\right]},
$$

where $V_{i}\left(l, x_{i, \tau, r} ; \theta\right), l=1, \ldots, L$ solve the value function iteration in (2) and (3). The second part of the likelihood can be determined through a parametric version of the relation in Theorem 3.1:

$$
F_{a}^{(r)}(\theta)=F_{a}\left(\theta_{g}\right)\left(\sum_{l=1}^{L} \Lambda_{l}(\theta) F_{l}\left(\theta_{g}\right)\right)^{r-1} . \forall a \in \mathcal{A}
$$

$F_{a}\left(\theta_{g}\right)$ collects the one period conditional transition probabilities parameterized by $\theta_{g}$, while the diagonal matrix $\Lambda_{l}(\theta)$ collects $\sigma\left(a_{i, \tau r} \mid x_{i, \tau r} ; \theta\right)$ given by the logistic formula above. The main computational challenge of this estimator is the need to nest the value function iteraction in (2) and (3) to calculate $\sigma\left(a_{i, \tau r} \mid x_{i, \tau r} ; \theta\right)$ as a function of the entire parameter vector $\theta=\left(\theta_{u}, \theta_{g}\right)$ during each likelihood evaluation.

\subsection{Estimation of Transition Matrices}

With complete data $D$, Rust (1987) also suggested a two step procedure in which $\theta_{g}$ is first estimated by maximizing $\ell^{M T P}\left(\theta_{g} \mid D_{r}\right)$. Conditional on $\hat{\theta}_{g}, \ell^{C C P}\left(\theta_{u}, \theta_{g} \mid D\right)$ is maximized with respect to $\theta_{u}$. While the incomplete data $D_{r}$ likelihood $\ell^{M T P}\left(\theta \mid D_{r}\right)$ is not separable in $\theta_{u}$ and $\theta_{g}$, a variant of this two step approach is feasible by viewing $\ell^{M T P}\left(\theta \mid D_{r}\right)$ as a function of $\theta_{g}$ and an initial estimate of the unconditional transition matrix $T$. For $F_{a}=$ $\left[g\left(x_{t+1} \mid x_{t}, a_{t}=a\right)\right]:$

$$
F_{a}^{(r)}\left(\theta_{g}, T\right)=F_{a}\left(\theta_{g}\right)[T]^{r-1}
$$

In the first step, an estimate of $\hat{T}$ is estimated using the likelihood function (11) in section 3.1. In step two, $\ell^{M T P}\left(\theta \mid D_{r}\right)$, as a function of $\theta_{g}$ and $T$, is maximized with respect to $\theta_{g}$ taking 
$\hat{T}$ as given. The knowledge of $\hat{\theta}_{g}$ allows for the iterations in (2) and (3) for each $\theta_{u}$. Finally, given $\hat{\theta}_{g}, \hat{\theta}_{u}$ is obtained by optimizing $\ell^{C C P}\left(\theta \mid D_{r}\right)$. In multi-step methods, standard errors need to be corrected to account for the impact of initial stage estimation. Alternatively, multiple step estimates can be used as starting values for the nested likelihood method. The separation of $\theta_{u}$ and $\theta_{g}$ reduces computation burden, but nesting value function iterations within likelihood evaluation is still computationally difficult.

Alternatively, one can abandon a parametric specification for $F_{a}\left(\theta_{g}\right)$ and recover it as an implied function of $F_{a}^{(r)}$ and $[T]$, as

$$
F_{a}=F_{a}^{(r)}\left([T]^{r-1}\right)^{-1}
$$

where both $F_{a}^{(r)}$ and $\hat{T}$ can be estimated using the likelihood function (11) in section 3.1. Each of the methods in this section can be readily generalized to allow for continuous state variables.

\subsection{Multi-step Semiparametric Estimators}

An insight developed by Hotz and Miller (1993) and extensively explored by the recent literature including Aguirregabiria and Mira (2002), Pesendorfer and Schmidt-Dengler (2010), Bajari, Benkard, and Levin (2007) and Bajari, Chernozhukov, Hong, and Nekipelov (2009), is that nested value function iteration can be replaced by a single step of nonparametric conditional choice probability estimation, which can be inverted to recover the value functions.

In essence, these methods show that given knowledge of the discount rate $\beta$, and given that the conditional choice probabilities $\sigma(a \mid x)$ can be flexibly estimated from the data, the choice specific value functions can be expressed as a function of the parametric static utilities $\Pi_{i}\left(a_{i}, x ; \theta_{u}\right)$, the nonparametric estimates of $\sigma(a \mid x)$ and the Markov transition density $g\left(x_{i, t+1} \mid x_{i, t}, a_{i, t} ; \theta_{g}\right)$. The general relation is given by,

$$
V_{i}\left(a_{i}, s ; \theta_{u}, \theta_{g}\right)=\Pi_{i}\left(a_{i}, s ; \theta_{u}\right)-\beta E\left[\log \sigma_{i}\left(a_{j} \mid s^{\prime}\right) \mid s, a_{i}\right]+\beta E\left[V_{i}\left(a_{j}, s^{\prime}\right) \mid s, a_{i}\right]
$$

For each $\theta$, the implied values of $V_{i}\left(a_{i}, s ; \theta\right)$ can be used to construct an alternative estimate of $\sigma(a \mid x)$, which can be compared to the original estimate to generate a parameter estimate for $\theta_{u}$. In the discrete state variable model that we considered, this can be represented as

$$
V_{a}(\theta)=\Pi_{a}\left(\theta_{u}\right)-\beta F_{a} \Lambda_{a}+\beta F_{a} V_{a}(\theta),
$$


leading to a solution that

$$
V_{a}(\theta)=\left(I-\beta F_{a}\right)^{-1}\left(\Pi_{a}\left(\theta_{u}\right)-\beta F_{a} \log \Lambda_{a}\right)
$$

This can be used to form a pseudo likelihood function for $\theta_{u}$ based on $\ell^{C C P}\left(\theta \mid D_{r}\right)$,

$$
\ell^{P L}\left(\theta_{u}\right)=\sum_{i=1}^{n} \sum_{\tau=0}^{M}\left(\log \exp V_{a_{i \tau}}\left(\theta_{u}\right)-\log \sum_{l=1}^{L} \exp V_{a_{l}}\left(\theta_{u}\right)\right) .
$$

Since it also holds that $V_{a}-V_{0}=\log \Lambda_{a}-\log \Lambda_{0}$, Hotz and Miller (1993) suggests an estimation procedure that chooses $\theta_{u}$ to minimize the difference

$$
\log \Lambda_{a}-\log \Lambda_{0}-\left(\left(I-\beta F_{a}\right)^{-1}\left(\Pi_{a}\left(\theta_{u}\right)-\beta F_{a} \log \Lambda_{a}\right)-\left(I-\beta F_{0}\right)^{-1}\left(\Pi_{0}\left(\theta_{u}\right)-\beta F_{0} \log \Lambda_{0}\right)\right)
$$

in the form of a nonlinear regression. When the period utility functions are linearly specified: $\Pi_{a}\left(\theta_{u}\right)=Z_{a}^{\prime} \theta_{u}$, where $Z_{a}=(z(a, x), \forall x)$ is the vector of regressions of $\operatorname{dim}(X)$, this can be implemented as a linear regression of $\log \Lambda_{a}-\log \Lambda_{0}+\beta\left(I-\beta F_{a}\right)^{-1} F_{a} \log \Lambda_{a}-$ $\beta\left(I-\beta F_{0}\right)^{-1} F_{0} \log \Lambda_{0}$ on $\left(I-\beta F_{a}\right)^{-1} Z_{a}-\left(I-\beta F_{0}\right)^{-1} Z_{0}$. In this case the pseudo likelihood function is a linear logit model, where $V_{a_{i \tau}}\left(\theta_{u}\right)=\left(I-\beta F_{a}\right)^{-1}\left(Z_{a}^{\prime} \theta_{u}-\beta F_{a} \log \Lambda_{a}\right)$.

A key component in the pseudo-likelihood method in Hotz and Miller (1993) is the estimation of $F_{a}$. This follows similar methods discussed in section 4.1, as in equation (15).

\subsection{Large Sample Distributions}

The large sample distributions of the estimators discuss above, which adapt previous DDCM estimation methods developed by Rust (1987), Hotz and Miller (1993) and many others to accommodate time aggregated data, can be derived using methods that are well known from the literature. Since these results are standard, in the following we provide a brief summary.

For both the nested fixed point estimator and the multi-step estimators, the large sample distributions can be obtained by applying multi-step MLE theory, which includes one-step estimators as special cases. The first stage maximum likelihood estimators of $\hat{f}_{a}^{(r)}$ and $\hat{T}$ usually follow an asymptotic normal distribution.

$$
\sqrt{N}\left(\hat{f}^{(r)}\left(x_{t+r} \mid x_{t}, a_{t}\right)-f^{(r)}\left(x_{t+r} \mid x_{t}, a_{t}\right)\right) \stackrel{D}{\rightarrow} N\left(0, \mathcal{V}_{f}^{r}\right)
$$

where $N=n M$ and similarly

$$
\sqrt{N}\left(\hat{T}\left(x_{t+r} \mid x_{t}\right)-T\left(x_{t+r} \mid x_{t}\right)\right) \stackrel{D}{\rightarrow} N\left(0, \mathcal{V}_{T}\right)
$$


For $g=H\left(f^{r}, T\right)$, by the functional invariability property of the ML theory, $\hat{g}$ also has the asymptotic normality

$$
\sqrt{N}\left(\hat{g}\left(x_{t+1} \mid x_{t}, a_{t}\right)-g\left(x_{t+1} \mid x_{t}, a_{t}\right)\right) \stackrel{D}{\rightarrow} N\left(0, \mathcal{V}_{g}\right)
$$

where $V_{g}$ can be obtained by the delta method as

$$
\mathcal{V}_{g}=\left(\frac{\partial H}{\partial f^{r}}, \frac{\partial H}{\partial T}\right)^{\prime} \operatorname{diag}\left(\mathcal{V}_{f^{r}}, \mathcal{V}_{T}\right)\left(\frac{\partial H}{\partial f^{r}}, \frac{\partial H}{\partial T}\right) .
$$

Next, using the theory of multi-step estimators (see for example Newey and McFadden (1994) and Murphy and Topel (2002) among others), $\theta_{u}$ also follows an asymptotic normality distribution. The asymptotic covariance matrix for $\theta_{u}$ that accounts from the estimation uncertainty from previous steps is given by

$$
\mathcal{V}_{\theta}=\frac{1}{N}\left[\mathcal{V}_{2}+\mathcal{V}_{2}\left(C \mathcal{V}_{g} C^{\prime}-R \mathcal{V}_{g} C^{\prime}-C \mathcal{V}_{g} R^{\prime}\right) \mathcal{V}_{2}\right] .
$$

In the above, $\mathcal{V}_{2}=A s y \cdot \operatorname{Var}\left[\sqrt{N}\left(\hat{\theta}_{u}-\theta_{u}\right)\right]$ is the asymptotic variance of $\hat{\theta}_{u}$ based on the $\log$-likelihood $\ell_{2}=\ell\left(\theta_{u}, \theta_{g} \mid D_{r}\right)$ that treats $\theta_{g}$ as known. The other components in $\mathcal{V}_{\theta}$ include

$$
C=E\left[\frac{1}{N}\left(\frac{\partial \ell_{2}}{\partial \theta_{u}}\right)\left(\frac{\partial \ell_{2}}{\partial \theta_{g}^{\prime}}\right)\right]
$$

and

$$
R=E\left[\frac{1}{N}\left(\frac{\partial \ell_{2}}{\partial \theta_{u}}\right)\left(\frac{\partial \ell^{M T P}}{\partial \theta_{g}^{\prime}}\right)\right]
$$

. Each of the components in $\mathcal{V}_{\theta}$ can be estimated consistently using sample analogs:

$$
\begin{gathered}
\hat{\mathcal{V}}_{2}=\left[\frac{1}{N} \sum_{i=1}^{n} \sum_{\tau=0}^{M}\left(\frac{\partial \log f_{2 i \tau}}{\partial \hat{\theta}_{u}}\right)\left(\frac{\partial \log f_{2 i \tau}}{\partial \hat{\theta}_{u}^{\prime}}\right)\right]^{-1} \\
\hat{C}=\frac{1}{N} \sum_{i=1}^{n} \sum_{\tau=0}^{M}\left(\frac{\partial \log f_{2 i \tau}}{\partial \hat{\theta}_{u}}\right)\left(\frac{\partial \log f_{2 i \tau}}{\partial \hat{\theta}_{g}^{\prime}}\right)
\end{gathered}
$$

and

$$
\hat{R}=\frac{1}{N} \sum_{i=1}^{n} \sum_{\tau=0}^{M}\left(\frac{\partial \log f_{2 i \tau}}{\partial \hat{\theta}_{u}}\right)\left(\frac{\partial \log f_{i \tau}}{\partial \hat{\theta}_{g}^{\prime}}\right) .
$$

In the above, $\log f_{2}=\log \sigma\left(\theta_{u} \mid D_{r}\right)+\log f\left(\hat{\theta}_{g} \mid D_{r}\right)$. In particular, Aguirregabiria and Mira (2002) showed that the efficiency of the nested fixed point MLE can be achieved by iterating the partial MLE method. Alternatively, instead of estimating the asymptotic variance matrix analytically, it is also possible to conduct inference using resampling methods, or by combining analytic estimation with resampling methods (e.g. Armstrong, Bertanha, and Hong (2014)). 


\section{Monte Carlo Simulation}

\subsection{A Numerical Simulation Example}

In this section we present an interesting finding regarding the statistical efficiency of estimating the transition matrix. A prior, we expect less accuracy in an indirect estimate of the one period transition matrix recovered from $r$ period time aggregated data than in a direct estimate when single period disaggregate data is available. Surprisingly, this is not necessarily the case. The efficiency ranking can be reversed. In the following we will use a simple 2 by 2 example to illustrate this possibility. Suppose

$$
A=\left[\begin{array}{cc}
x & 1-x \\
1-y & y
\end{array}\right], \quad B=A^{r}=\left[\begin{array}{cc}
a & 1-a \\
1-b & b
\end{array}\right]
$$

We are interested in estimating $(x, y)$ and $(a, b)$. If we estimate $x$ and $y$ directly, in large sample we have $\hat{x} \sim N\left(x, \frac{x(1-x)}{N_{1}}\right)$ and $\hat{y} \sim N\left(y, \frac{y(1-y)}{N_{2}}\right) . N_{1}$ and $N_{2}$ are the numbers of observations initially in state 1 and state 2 , respectively. Similarly, if we estimate from $r^{\text {th }}$ period data, we have $\hat{a} \sim N\left(a, \frac{a(1-a)}{N_{1}}\right)$ and $\hat{b} \sim N\left(b, \frac{b(1-b)}{N_{2}}\right)$. It turns out that given the true values of $a$ and $b$, the variances of $\hat{a}$ and $\hat{b}$ do not depend on the period $r$. To solve analytically, consider $y=1$, where

$$
\left[\begin{array}{cc}
x & 1-x \\
0 & 1
\end{array}\right]^{r}=\left[\begin{array}{cc}
x^{r} & 1-x^{r} \\
0 & 1
\end{array}\right]=\left[\begin{array}{cc}
a & 1-a \\
0 & 1
\end{array}\right]
$$

On the one hand, the variance of the direct one period estimator is: $\operatorname{Var}(\hat{x})=\frac{x(1-x)}{N_{1}}$. On the other hand, the variance of the estimator of $a$ is: $\operatorname{Var}(\hat{a})=\frac{a(1-a)}{N_{1}}=\frac{x^{r}\left(1-x^{r}\right)}{N_{1}}$. An indirect estimate $\bar{x}$ of $x$ is given by $r^{t h}$ root of $\hat{a}: \bar{x}=\sqrt[r]{\hat{a}}$. By the Delta method, $\operatorname{Var}(\bar{x})=\left(\frac{1}{r} a^{\frac{1}{r}-1}\right)^{2} \operatorname{Var}(\hat{a})=\frac{x^{2-r}\left(1-x^{r}\right)}{r^{2} N_{1}}$. The ratio of these two variances is:

$$
\frac{\operatorname{Var}(\bar{x})}{\operatorname{Var}(\hat{x})}=\frac{x^{1-r}\left(1-x^{r}\right)}{r^{2}(1-x)}=\frac{1}{r^{2}}\left[1+\frac{1}{x}+\frac{1}{x^{2}}+\ldots++\frac{1}{x^{r-2}}+\frac{1}{x^{r-1}}\right] .
$$

The following table shows the threshold values of $x$ under different values of $r$, above which the variance of the indirect estimate is lower than that of the direct estimate.

Table 1 about here. 
When value of $x$ is larger than the threshold value, indirect estimation is more efficient than direct estimation. The threshold value increases with the length of time period $r$, shown in the following graph:

Figure 2 about here.

In addition, we also compare the variances of indirect estimates among aggregate data with different values of $r$. The graph below indicates that the variances vary substantially with different probability values of $x$ and different time periods of $r$ (before being divided by $N_{1}$ ). It can be seen that different values of $x$ correspond to different "optimal" data observation periods when the asymptotic variance is minimized.

Figure 3 about here.

The following table and graph show the optimal period values of $r$ for different values of probability $x$.

Table 2 about here.

Figure 4 about here.

In practice, if there are budget constraints that limit data collection to only two periods, the previous table is important for providing guidance to researchers when they choose the optimal time interval for efficient estimation.

However, it is more complicated for general cases, where $y \neq 1$. Analytical result is infeasible in these cases, but numerical solution is available. The threshold value will be value pairs of $(x, y)$, instead of a single value of $x$.

In Proposition 3.1, we have solved indirect estimation from aggregate data in general $2 \times 2$ cases. Unknown estimator of $\bar{x}$ and $\bar{y}$ are functions of $\hat{a}$ and $\hat{b}$. By Delta method, we know that ( $\hat{a}$ and $\hat{b}$ are independent):

$$
\operatorname{Var}(\bar{x})=\left(\frac{\partial x(\hat{a}, \hat{b})}{\partial a}\right)^{2} \operatorname{Var}(\hat{a})+\left(\frac{\partial x(\hat{a}, \hat{b})}{\partial b}\right)^{2} \operatorname{Var}(\hat{b})
$$


Since it is not easy to get analytical partial derivatives of function $\bar{x}=x(\hat{a}, \hat{b})$, we do numerical calculation for variances of $\bar{x}$. We only consider cases that $a+b>1$ and $x+y>1$, where unique solution is ensured. In addition, the numbers of observations in state 1 and state 2 initially are supposed to be the same. We evaluate $(x, y)$ with different values, thus variance ratios of estimator $\bar{x}$ when $r=2$ and estimator $\hat{x}$ when $r=1$ are shown in the following table:

Table 3 about here.

In the lower right corner, values that are less than 1 indicate that indirect estimation with $r=2$ data is more efficient than direct estimation with $r=1$. In this way, we can find threshold value pairs of $(x, y)$, where variance ratios are exactly equal to 1 . These threshold value pairs are shown as the red curve in the following graph:

Figure 5 about here.

Similarly, we also compare the variances of indirect estimates among aggregate data with different values of $r$. As a result, the "optimal" data observation period is obtained for a given value pair of $(x, y)$, as shown in the graph below:

Figure 6 about here.

Meanwhile, result from simulation strongly supports our theoretical analysis. We consider cases where $r=2$ :

$$
\left[\begin{array}{cc}
x & 1-x \\
1-y & y
\end{array}\right]^{2}=\left[\begin{array}{cc}
a & 1-a \\
1-b & b
\end{array}\right]
$$

Each time, 10,000 observations are simulated, where $N_{1}=N_{2}=5,000$. We collect the direct estimation of $\hat{x}$ from disaggregate data, and indirect estimation of $\bar{x}$ from aggregate data. We repeat the simulation 10,000 times and calculate the average square deviations of $\hat{x}$ and $\bar{x}$. Their ratios are in the following table (values in brackets are ratios of theoretical variances from the previous table):

Table 4 about here. 


\subsection{Dynamic Discrete Choice Model Simulation}

Below are the numerical simulation results of four cases in the context of Rust's engine replacement problem: a. Rust method with original data $D$; b. Hotz-Miller method with original data $D$; c. Rust method with aggregated data $D_{r}$; d. Hotz-Miller method with original data $D_{r}$.

For the configurations, it is set that $r=2,|\mathcal{A}|=2$ and $|\mathcal{X}|=3$. The discount factor $\beta=0.95$, the number of agents is 50 and the observational time horizon $T=100$. The true parameter $\theta$ is $\theta=\left(\theta_{u}, \theta_{g}\right)$ where $\theta_{u}=4.5$ and $\theta_{g}$ is given via $g$ as below:

$$
\begin{gathered}
g\left(x^{\prime} \mid x, a=0\right)=\left(\begin{array}{lll}
0.82675634 & 0.01760606 & 0.1556376 \\
0.03157846 & 0.83906271 & 0.1293588 \\
0.04623463 & 0.02309968 & 0.9306657
\end{array}\right) \\
g\left(x^{\prime} \mid x, a=1\right)=\left(\begin{array}{lll}
0.793063211 & 0.1130186 & 0.09391818 \\
0.011898949 & 0.8901177 & 0.09798333 \\
0.005132359 & 0.1810372 & 0.81383047
\end{array}\right) .
\end{gathered}
$$

The utility function $U(a, x)$ is given as

$$
U(a, x)=(a-1) \cdot(x+1)-a \cdot \theta_{u}
$$

in which the action variable $a$ can take value 0 or 1 , denoting whether or not the engine is replaced. The observed state variable $x$ can take value 0,1 or 2 . Intuitively, $(a-1) \cdot(x+1)$ represents the engine maintenance cost while $\theta$ represents the engine replacement cost.

Then, 1000 simulations are conducted under the classical Rust's model assumptions. Since $r=2$, only the odd number of the simulated data entries are taken as observed ones. Afterwards, 1000 pairs of estimators $\hat{\theta}_{\text {Rust }}$ and $\hat{\theta}_{H M}$ are computed with $D_{r}$ by using the time aggregated estimation methodologies introduced in this paper. For comparison, another 1000 pairs of estimators $\bar{\theta}_{R u s t}$ and $\bar{\theta}_{H M}$ are computed with $D$ by using the original Rust and Hotz-Miller methods.

The results are collected as follows. First, as the estimations of $g$ under Rust and pseudo MLE based Hotz-Miller methods are no different, $\hat{g}$ and $\bar{g}$ are obtained and summarized in the following two tables. One note to take is that in practice, sometimes there are some 
small imaginary parts introduced as numerical errors when $\hat{g}$ are calculated; to mitigate this effect, the final $\hat{g}$ are rounded against the imaginary parts and are normalized so that finally it is a collection of real stochastic matrices.

Table 5 about here.

Table 6 about here.

Next the estimations of the replacement $\operatorname{cost} \theta_{u}$ under four different settings are demonstrated below:

Table 7 about here.

To visualize these results, some plots are made below.

Figure 7 about here.

Figure 8 about here.

\section{Concluding Remarks}

In this paper we study the use of time aggregated data on the estimation of dynamic discrete models. We find that in the reduced form model, indirect estimation using time aggregated data can lead to efficiency gains. This has implications for both quasi maximum likelihood estimator and limited information maximum likelihood estimators.

It is worth noting that in the reduced form model, the conditional choice probabilities and the multi-period state transition probability matrix exactly identify the single-period state transition probability matrix, which leaves no additional degree of freedom to generate testable for the purpose of selecting among multiple stochastic roots. This might be possible, however, in the structural model when researchers are willing to impose additional restrictions on different components of the model. For example, if there are variables that enter only the state transition probability matrix but not the static utility function (an assumption that has been employed to identify discount rates, as e.g. in Magnac and Thesmar (2002), Aguirregabiria and Mira (2010) and Arcidiacono and Miller (2014)), then it might be 
possible to choose the stochastic root in such a way that the recovered static utility function in the structural model depends minimally on the excluded variables that only shift the state transition probability matrix.

\section{References}

Aguirregabiria, V., And P. Mira (2002): "Swapping the nested fixed point algorithm: a class of estimators for discrete Markov decision models," Econometrica, 70, 1519-1543.

$$
\text { (2007): "Sequential estimation of dynamic discrete games," Econometrica, 75, } 1 .
$$

Aguirregabiria, V., And P. Mira (2010): "Dynamic discrete choice structural models: A survey," Journal of Econometrics, 156(1), 38-67.

Arcidiacono, P., P. Bayer, J. R. Blevins, and P. B. Ellickson (2012): "Estimation of dynamic discrete choice models in continuous time," Discussion paper, National Bureau of Economic Research.

Arcidiacono, P., And J. B. Jones (2003): "Finite mixture distributions, sequential likelihood and the EM algorithm," Econometrica, 71(3), 933-946.

Arcidiacono, P., And R. A. Miller (2011): "Conditional choice probability estimation of dynamic discrete choice models with unobserved heterogeneity," Econometrica, 79(6), 18231867.

(2014): "Identifying Dynamic Discrete Choice Models off Short Panels," Duke University and Carnegie Mellon University.

Armstrong, T. B., M. Bertanha, And H. Hong (2014): "A fast resample method for parametric and semiparametric models," Journal of Econometrics, 179(2), 128-133.

Bajari, P., C. Benkard, And J. Levin (2007): "Estimating Dynamic Models of Imperfect Competition," Econometrica, 75, 1331-1370.

Bajari, P., V. Chernozhukov, H. Hong, and D. Nekipelov (2009): "Nonparametric and semiparametric analysis of a dynamic game model," .

Berry, S., A. Pakes, And M. Ostrovsky (2003): "Simple estimators for the parameters of dynamic games (with entry/exit examples)," Technical Report, Harvard University.

Blevins, J. R. (2013): "Identifying Restrictions for Finite Parameter Continuous Time Models with Discrete Time Data," Working Paper 13-01, Ohio State University.

(2014): "Identification and Estimation of Continuous Time Dynamic Games," Working paper, Ohio State University.

CArdoso, J. (2012): "Computation of the matrix pth root and its Frechet derivative by integrals," Electronic Transactions on Numerical Analysis, 39, 414-436. 
Doraszelski, U., AND K. L. JudD (2012): "Avoiding the curse of dimensionality in dynamic stochastic games," Quantitative Economics, 3(1), 53-93.

FANG, H., AND Y. WANG (2010): "Estimating dynamic discrete choice models with hyperbolic discounting, with an application to mammography decisions," Discussion paper, National Bureau of Economic Research.

He, Q., And E. Gunn (2003): "A note on the stochastic roots of stochastic matrices," J. System Sci. and System Eng., 12(2), 210-223.

Hotz, J., And R. Miller (1993): "Conditional Choice Probabilties and the Estimation of Dynamic Models," Review of Economic Studies, 60, 497-529.

Hu, Y., AND M. Shum (2012): "Nonparametric identification of dynamic models with unobserved state variables," Journal of Econometrics, 171(1), 32-44.

Imai, S., N. Jain, AND A. Ching (2009): "Bayesian estimation of dynamic discrete choice models," Econometrica, 77, 1865-1899.

Jenkins, M., P. Liu, D. McFadden, And R. Matzkin (2004): "The Browser War: Econometric Analysis of Markov Perfect Equilibrium in Markets with Network Effects," UC Berkeley, working paper.

Kasahara, H., And K. Shimotsu (2008): "Nonparametric identification of finite mixture models of dynamic discrete choices," Econometrica, 77, 135-176.

Lin, L. (2011): "Roots of Stochastic Matrices and Fractional Matrix Powers (2011)," PhD thesis, Manchester Institute for Mathematical Sciences.

Magnac, T., And D. Thesmar (2002): "Identifying dynamic discrete decision processes," Econometrica, 70(2), 801-816.

McDonald, J. J., P. P., And M. J. Tsatsomeros (2013): "Matrix roots of eventually positive matrices," Linear Algebra and its Applications, available online 7 December 2013.

Murphy, K. M., And R. H. Topel (2002): "Estimation and inference in two-step econometric models," Journal of Business \& Economic Statistics, 20(1), 88-97.

Newey, W., And D. McFadden (1994): "Large Sample Estimation and Hypothesis Testing," in Handbook of Econometrics, Vol. 4, ed. by R. Engle, and D. McFadden, pp. 2113-2241. North Holland.

Norets, A. (2009): "Inference in Dynamic Discrete Choice Models With Serially orrelated Unobserved State Variables," Econometrica, 77, 1665-1682.

Pesendorfer, M., And P. Schmidt-Dengler (2010): "Sequential estimation of dynamic discrete games: A comment," Econometrica, 78, 833-842.

Rust, J. (1987): "Optimal Replacement of GMC Bus Engines: An Empirical Model of Harold Zurcher," Econometrica, 55, 999-1033.

RyAN, S. P. (2012): "The costs of environmental regulation in a concentrated industry," Econometrica, 80(3), 1019-1061. 


\section{A Proof of proposition 1}

First, we need to decompose the unknown matrix:

$$
\left[\begin{array}{cc}
x & 1-x \\
1-y & y
\end{array}\right]=\left[\begin{array}{cc}
1 & 1-x \\
1 & -1+y
\end{array}\right]\left[\begin{array}{cc}
1 & 0 \\
0 & x+y-1
\end{array}\right]\left[\begin{array}{cc}
1 & 1-x \\
1 & -1+y
\end{array}\right]^{-1}
$$

Thus,

$$
\left[\begin{array}{cc}
x & 1-x \\
1-y & y
\end{array}\right]^{r}=\left[\begin{array}{cc}
1 & 1-x \\
1 & -1+y
\end{array}\right]\left[\begin{array}{cc}
1 & 0 \\
0 & (x+y-1)^{r}
\end{array}\right]\left[\begin{array}{cc}
1 & 1-x \\
1 & -1+y
\end{array}\right]^{-1}
$$

Substitute the above equation into the original equation:

$$
\left[\begin{array}{cc}
\frac{-1+y-(1-x)(x+y-1)^{r}}{x+y-2} & \frac{x-1+(1-x)(x+y-1)^{r}}{x+y-2} \\
\frac{-1+y+(1-y)(x+y-1)^{r}}{x+y-2} & \frac{x-1-(1-y)(x+y-1)^{r}}{x+y-2}
\end{array}\right]=\left[\begin{array}{cc}
a & 1-a \\
1-b & b
\end{array}\right]
$$

Take the difference of the two elements of the first column in Equation 21:

$$
(x+y-1)^{r}=a+b-1
$$

Take the ratio of the right-upper and left-lower elements in Equation 21:

$$
\frac{1-x}{1-y}=\frac{1-a}{1-b}
$$

From these two important results in Equation 22 and Equation 23, we can get the solution in Theorem 3.1 easily. Any solution with element out of interval [0,1] has been eliminated. Equation 23 tells us that the ratio of the probabilities off diagonal in a 2-2 case doesn't change with time period $r$. Equation 22 suggests that the difference of the probabilities of every column in a 2-2 case changes in terms of power $r$ with time period $r$, which is the same with the change of the stochastic matrix itself. These two relationships help us not only understand how the stochastic matrix evolves with time, but also solve a 2-2 problem. 
Table 1: Threshold values of $x$ under different $r$

\begin{tabular}{|c|cccccccc|}
\hline value of $r$ & 2 & 3 & 4 & 5 & 6 & 7 & 8 & 9 \\
\hline threshold of $x$ & 0.33 & 0.42 & 0.49 & 0.54 & 0.58 & 0.61 & 0.64 & 0.66 \\
\hline value of $r$ & 10 & 15 & 20 & 25 & 30 & 40 & 50 & 100 \\
\hline threshold of $x$ & 0.68 & 0.75 & 0.79 & 0.82 & 0.84 & 0.87 & 0.89 & 0.94 \\
\hline
\end{tabular}

Table 2: Optimal value sof $r$ for different probabilities $x$.

\begin{tabular}{cc|cc|cc} 
interval of $\mathrm{x}$ & best value of $\mathrm{r}$ & interval of $\mathrm{x}$ & best value of $\mathrm{r}$ & interval of $\mathrm{x}$ & best value of $\mathrm{r}$ \\
\hline$(0.000,0.333)$ & 1 & $(0.808,0.829)$ & 8 & $(0.896,0.902)$ & 15 \\
$(0.333,0.525)$ & 2 & $(0.829,0.845)$ & 9 & $(0.902,0.908)$ & 16 \\
$(0.525,0.633)$ & 3 & $(0.845,0.859)$ & 10 & $(0.908,0.913)$ & 17 \\
$(0.633,0.701)$ & 4 & $(0.859,0.871)$ & 11 & $(0.913,0.917)$ & 18 \\
$(0.701,0.748)$ & 5 & $(0.871,0.880)$ & 12 & $(0.917,0.922)$ & 19 \\
$(0.748,0.782)$ & 6 & $(0.880,0.889)$ & 13 & $(0.922,0.925)$ & 20 \\
$(0.782,0.808)$ & 7 & $(0.889,0.896)$ & 14 & $(0.925,0.929)$ & 21
\end{tabular}


Table 3: Variance ratio of two estimators

\begin{tabular}{|cc|cccccccc|}
\hline & & \multicolumn{8}{|c|}{ value of $x$} \\
\hline \multirow{4}{*}{ value of $y$} & 0.2 & 0.3 & 0.4 & 0.5 & 0.6 & 0.7 & 0.8 & 0.9 \\
\hline & 0.2 & - & - & - & - & - & - & - & 2.26 \\
& 0.3 & - & - & - & - & - & - & 4.44 & 1.34 \\
& 0.4 & - & - & - & - & - & 7.39 & 2.00 & 1.04 \\
& 0.6 & - & - & - & - & 10.99 & 2.78 & 1.36 & 0.88 \\
& 0.7 & - & - & - & 15.04 & 3.61 & 1.68 & 1.05 & 0.77 \\
& 0.8 & - & 22.27 & 4.83 & 4.37 & 1.97 & 1.20 & 0.87 & 0.69 \\
& 0.9 & 21.61 & 4.42 & 1.97 & 1.23 & 0.91 & 0.74 & 0.64 & 0.57 \\
\hline
\end{tabular}

Table 4: Variance ratio

\begin{tabular}{|c|c|c|c|c|c|c|}
\hline & 0.5 & \multicolumn{3}{|c|}{ value of $x$} & 0.9 \\
\hline \multirow{5}{*}{ value of $y$} & 0.5 & - & - & $2.97(2.78)$ & $1.39(1.36)$ & $0.95(0.88)$ \\
\hline & 0.6 & - & $3.76(3.61)$ & $1.66(1.68)$ & $1.17(1.05)$ & $0.71(0.77)$ \\
\hline & 0.7 & $4.94(4.37)$ & $1.98(1.97)$ & $1.12(1.20)$ & $0.87(0.87)$ & $0.69(0.69)$ \\
\hline & 0.8 & $2.21(2.12)$ & $1.38(1.29)$ & $0.90(0.93)$ & $0.76(0.74)$ & $0.64(0.63)$ \\
\hline & 0.9 & $1.12(1.23)$ & $0.90(0.91)$ & $0.79(0.74)$ & $0.68(0.64)$ & $0.54(0.57)$ \\
\hline
\end{tabular}


Table 5: Estimation of $\hat{g}$

\begin{tabular}{cccccc}
\hline Element of $\hat{\theta}$ & $25 \%$ & Median & Mean & $75 \%$ & S.D. \\
\hline$\hat{g}\left(x^{\prime}=0 \mid x=0, a=0\right)$ & 0.8182 & 0.8269 & 0.8265 & 0.8346 & 0.0122 \\
$\hat{g}\left(x^{\prime}=1 \mid x=0, a=0\right)$ & 0.0143 & 0.0175 & 0.0177 & 0.0212 & 0.0053 \\
$\hat{g}\left(x^{\prime}=2 \mid x=0, a=0\right)$ & 0.1473 & 0.1555 & 0.1558 & 0.1635 & 0.0112 \\
$\hat{g}\left(x^{\prime}=0 \mid x=1, a=0\right)$ & 0.0266 & 0.0314 & 0.0318 & 0.0369 & 0.0077 \\
$\hat{g}\left(x^{\prime}=1 \mid x=1, a=0\right)$ & 0.8284 & 0.8387 & 0.8384 & 0.8490 & 0.0151 \\
$\hat{g}\left(x^{\prime}=2 \mid x=1, a=0\right)$ & 0.1197 & 0.1288 & 0.1298 & 0.1391 & 0.0143 \\
$\hat{g}\left(x^{\prime}=0 \mid x=2, a=0\right)$ & 0.0420 & 0.0464 & 0.0466 & 0.0510 & 0.0065 \\
$\hat{g}\left(x^{\prime}=1 \mid x=2, a=0\right)$ & 0.0186 & 0.0235 & 0.0234 & 0.0283 & 0.0071 \\
$\hat{g}\left(x^{\prime}=2 \mid x=2, a=0\right)$ & 0.9235 & 0.9302 & 0.9300 & 0.9368 & 0.0094 \\
$\hat{g}\left(x^{\prime}=0 \mid x=0, a=1\right)$ & 0.6873 & 0.7853 & 0.7681 & 0.8722 & 0.1358 \\
$\hat{g}\left(x^{\prime}=1 \mid x=0, a=1\right)$ & 0.0498 & 0.1064 & 0.1220 & 0.1807 & 0.0927 \\
$\hat{g}\left(x^{\prime}=2 \mid x=0, a=1\right)$ & 0.0000 & 0.0827 & 0.1099 & 0.1798 & 0.1131 \\
$\hat{g}\left(x^{\prime}=0 \mid x=1, a=1\right)$ & 0.0000 & 0.0034 & 0.0215 & 0.0362 & 0.0303 \\
$\hat{g}\left(x^{\prime}=1 \mid x=1, a=1\right)$ & 0.8246 & 0.8819 & 0.8755 & 0.9372 & 0.0790 \\
$\hat{g}\left(x^{\prime}=2 \mid x=1, a=1\right)$ & 0.0409 & 0.0945 & 0.1029 & 0.1527 & 0.0770 \\
$\hat{g}\left(x^{\prime}=0 \mid x=2, a=1\right)$ & 0.0000 & 0.0039 & 0.0082 & 0.0138 & 0.0103 \\
$\hat{g}\left(x^{\prime}=1 \mid x=2, a=1\right)$ & 0.1610 & 0.1780 & 0.1806 & 0.1992 & 0.0287 \\
$\hat{g}\left(x^{\prime}=2 \mid x=2, a=1\right)$ & 0.7915 & 0.8127 & 0.8112 & 0.8320 & 0.0299 \\
\hline
\end{tabular}


Table 6: Estimation of $\bar{g}$

\begin{tabular}{cccccc}
\hline Element of $\bar{\theta}$ & $25 \%$ & Median & Mean & $75 \%$ & S.D. \\
\hline $\bar{g}\left(x^{\prime}=0 \mid x=0, a=0\right)$ & 0.8193 & 0.8268 & 0.8265 & 0.8336 & 0.0108 \\
$\bar{g}\left(x^{\prime}=1 \mid x=0, a=0\right)$ & 0.0152 & 0.0175 & 0.0176 & 0.0201 & 0.0038 \\
$\bar{g}\left(x^{\prime}=2 \mid x=0, a=0\right)$ & 0.1485 & 0.1559 & 0.1559 & 0.1626 & 0.0104 \\
$\bar{g}\left(x^{\prime}=0 \mid x=1, a=0\right)$ & 0.0281 & 0.0315 & 0.0318 & 0.0357 & 0.0058 \\
$\bar{g}\left(x^{\prime}=1 \mid x=1, a=0\right)$ & 0.8305 & 0.8395 & 0.8384 & 0.8466 & 0.0124 \\
$\bar{g}\left(x^{\prime}=2 \mid x=1, a=0\right)$ & 0.1219 & 0.1292 & 0.1297 & 0.1370 & 0.0113 \\
$\bar{g}\left(x^{\prime}=0 \mid x=2, a=0\right)$ & 0.0432 & 0.0463 & 0.0465 & 0.0496 & 0.0047 \\
$\bar{g}\left(x^{\prime}=1 \mid x=2, a=0\right)$ & 0.0210 & 0.0231 & 0.0232 & 0.0256 & 0.0033 \\
$\bar{g}\left(x^{\prime}=2 \mid x=2, a=0\right)$ & 0.9266 & 0.9303 & 0.9303 & 0.9344 & 0.0057 \\
\hline $\bar{g}\left(x^{\prime}=0 \mid x=0, a=1\right)$ & 0.7436 & 0.7895 & 0.7877 & 0.8333 & 0.0654 \\
$\bar{g}\left(x^{\prime}=1 \mid x=0, a=1\right)$ & 0.0797 & 0.1158 & 0.1171 & 0.1515 & 0.0532 \\
$\bar{g}\left(x^{\prime}=2 \mid x=0, a=1\right)$ & 0.0606 & 0.0909 & 0.0952 & 0.1256 & 0.0475 \\
$\bar{g}\left(x^{\prime}=0 \mid x=1, a=1\right)$ & 0.0000 & 0.0118 & 0.0119 & 0.0189 & 0.0124 \\
$\bar{g}\left(x^{\prime}=1 \mid x=1, a=1\right)$ & 0.8664 & 0.8913 & 0.8888 & 0.9136 & 0.0355 \\
$\bar{g}\left(x^{\prime}=2 \mid x=1, a=1\right)$ & 0.0746 & 0.0987 & 0.0994 & 0.1194 & 0.0334 \\
$\bar{g}\left(x^{\prime}=0 \mid x=2, a=1\right)$ & 0.0032 & 0.0050 & 0.0052 & 0.0069 & 0.0030 \\
$\bar{g}\left(x^{\prime}=1 \mid x=2, a=1\right)$ & 0.1709 & 0.1810 & 0.1814 & 0.1925 & 0.0162 \\
$\bar{g}\left(x^{\prime}=2 \mid x=2, a=1\right)$ & 0.8023 & 0.8142 & 0.8135 & 0.8249 & 0.0165 \\
\hline
\end{tabular}

Table 7: Estimation of replacement $\operatorname{cost} \theta_{u}$

\begin{tabular}{cccccc}
\hline Element of $\bar{\theta}$ & $25 \%$ & Median & Mean & $75 \%$ & S.D. \\
\hline$\hat{\theta}_{\text {uRust }}$ & 4.430 & 4.521 & 4.533 & 4.636 & 0.1481 \\
$\hat{\theta}_{u H M}$ & 4.432 & 4.523 & 4.530 & 4.623 & 0.1427 \\
$\bar{\theta}_{u \text { Rust }}$ & 4.453 & 4.502 & 4.503 & 4.550 & 0.0776 \\
$\bar{\theta}_{u H M}$ & 4.458 & 4.507 & 4.508 & 4.555 & 0.0777 \\
\hline
\end{tabular}


Figure 1: Illustration of Proposition 3.1

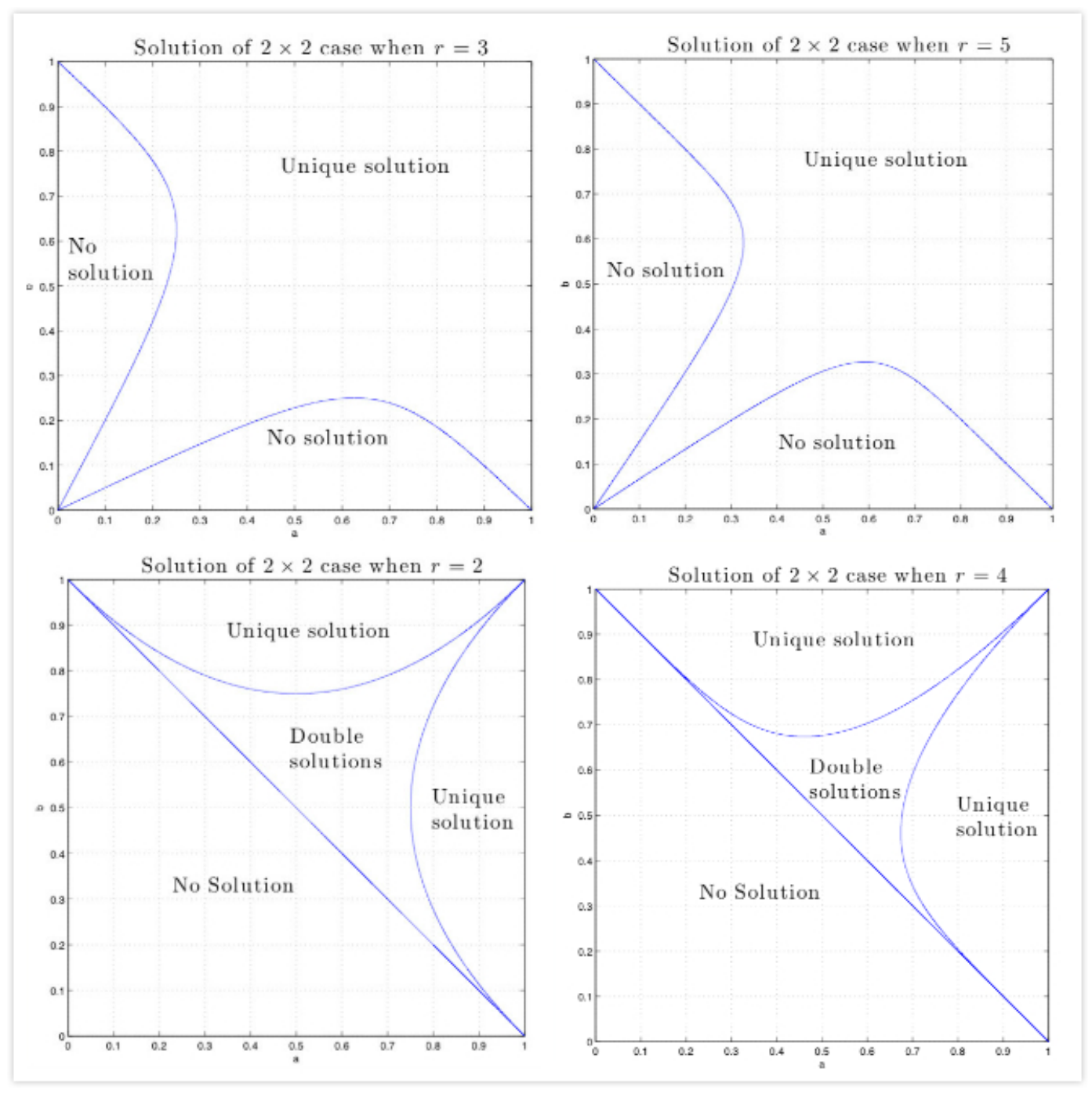


Figure 2: Threshold values of $x$ under different $r$

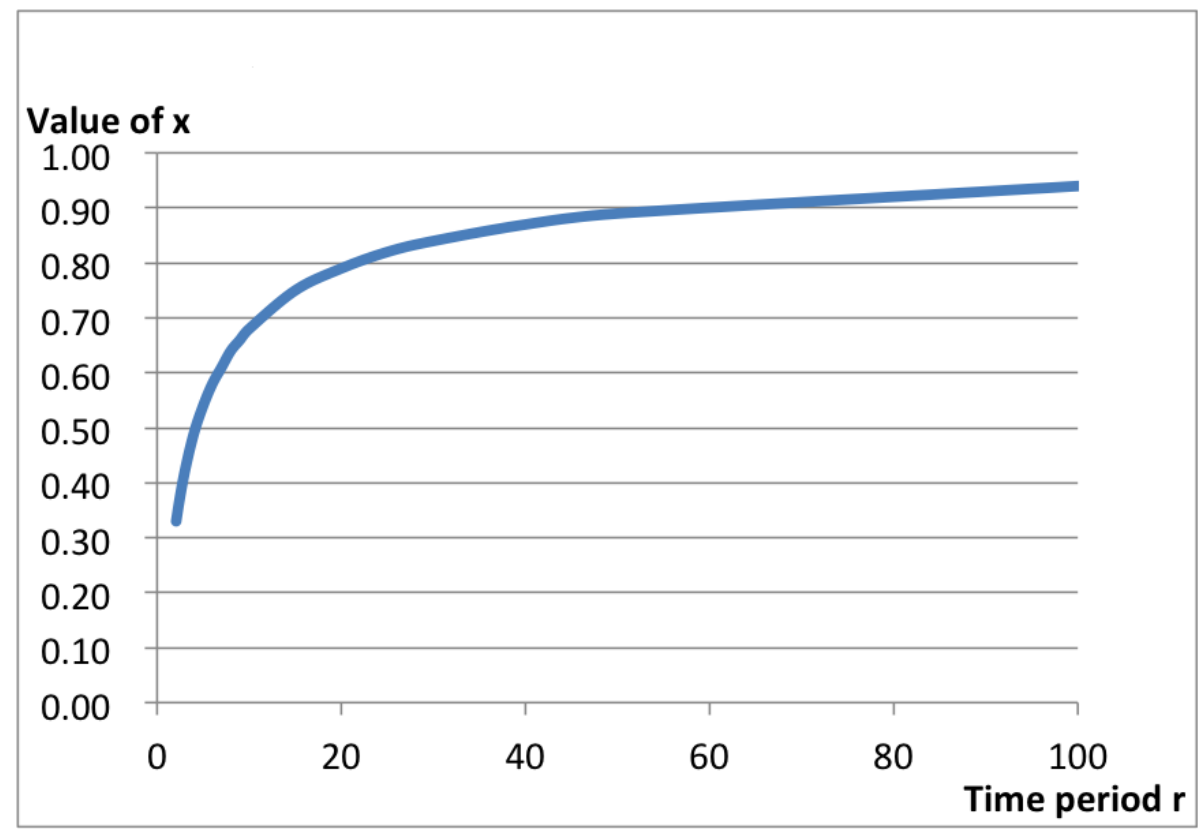

Figure 3: Variance comparison for different values of $r$

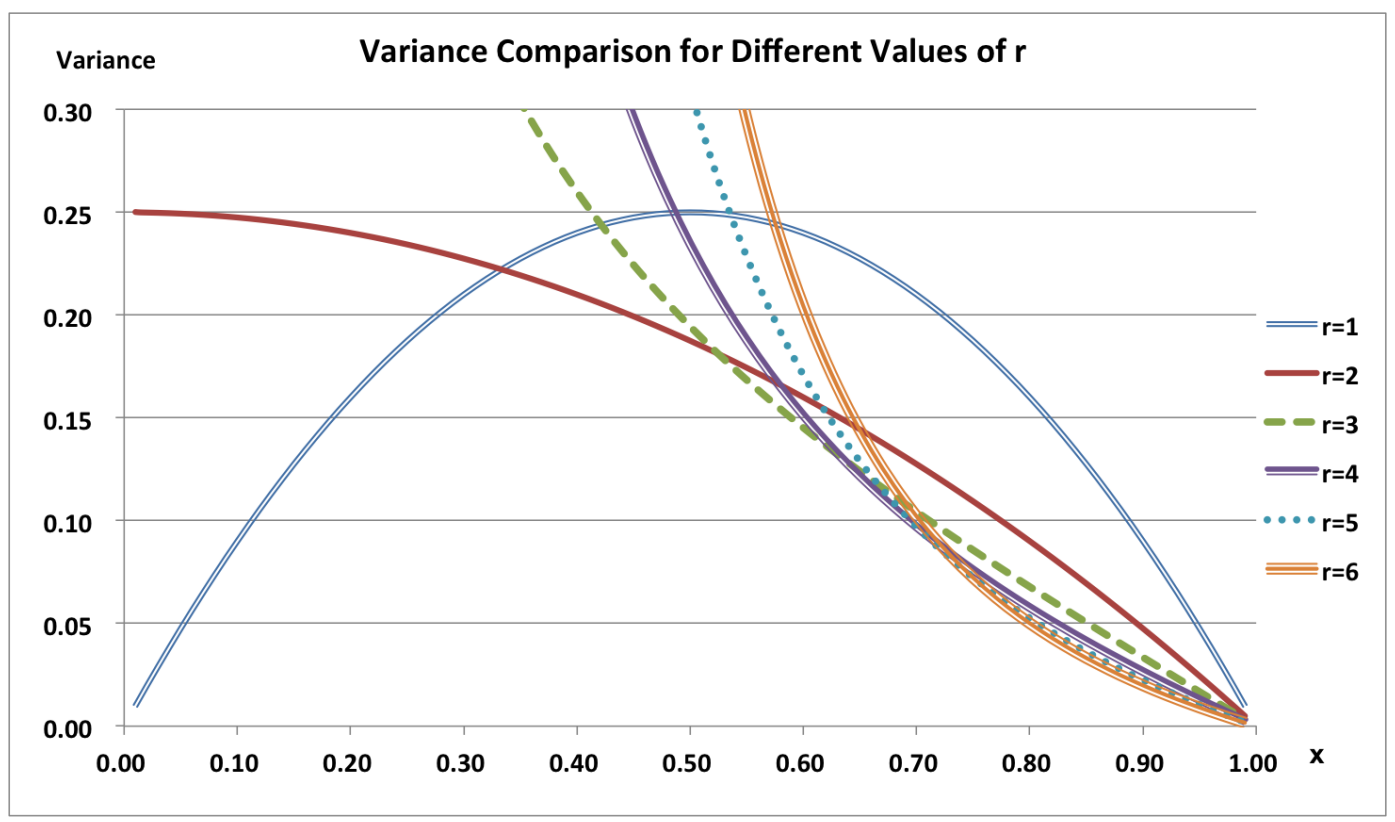


Figure 4: Best $r$ for $x$

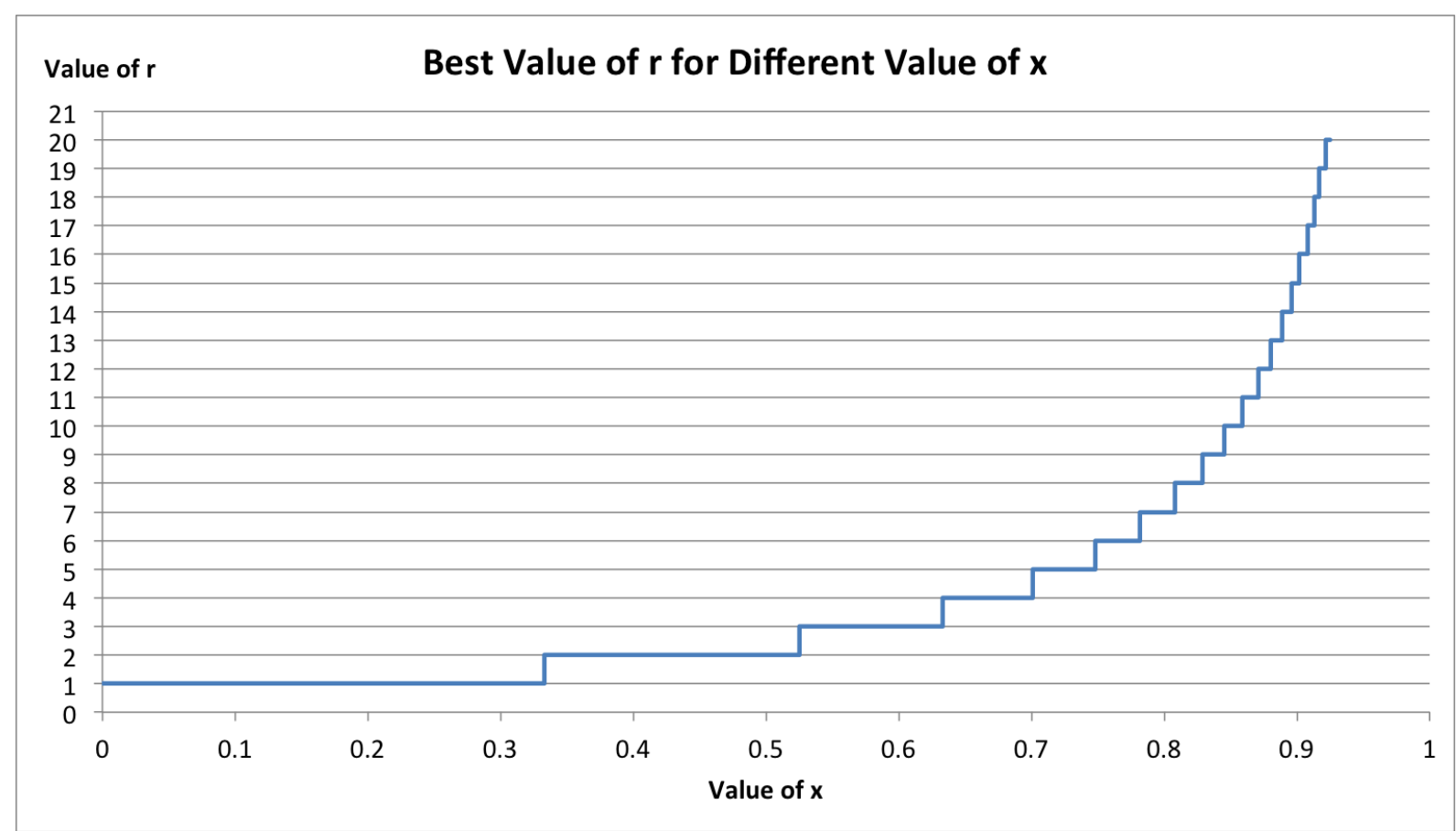


Figure 5: Variance compared with different $r$ across $x$ and $y$

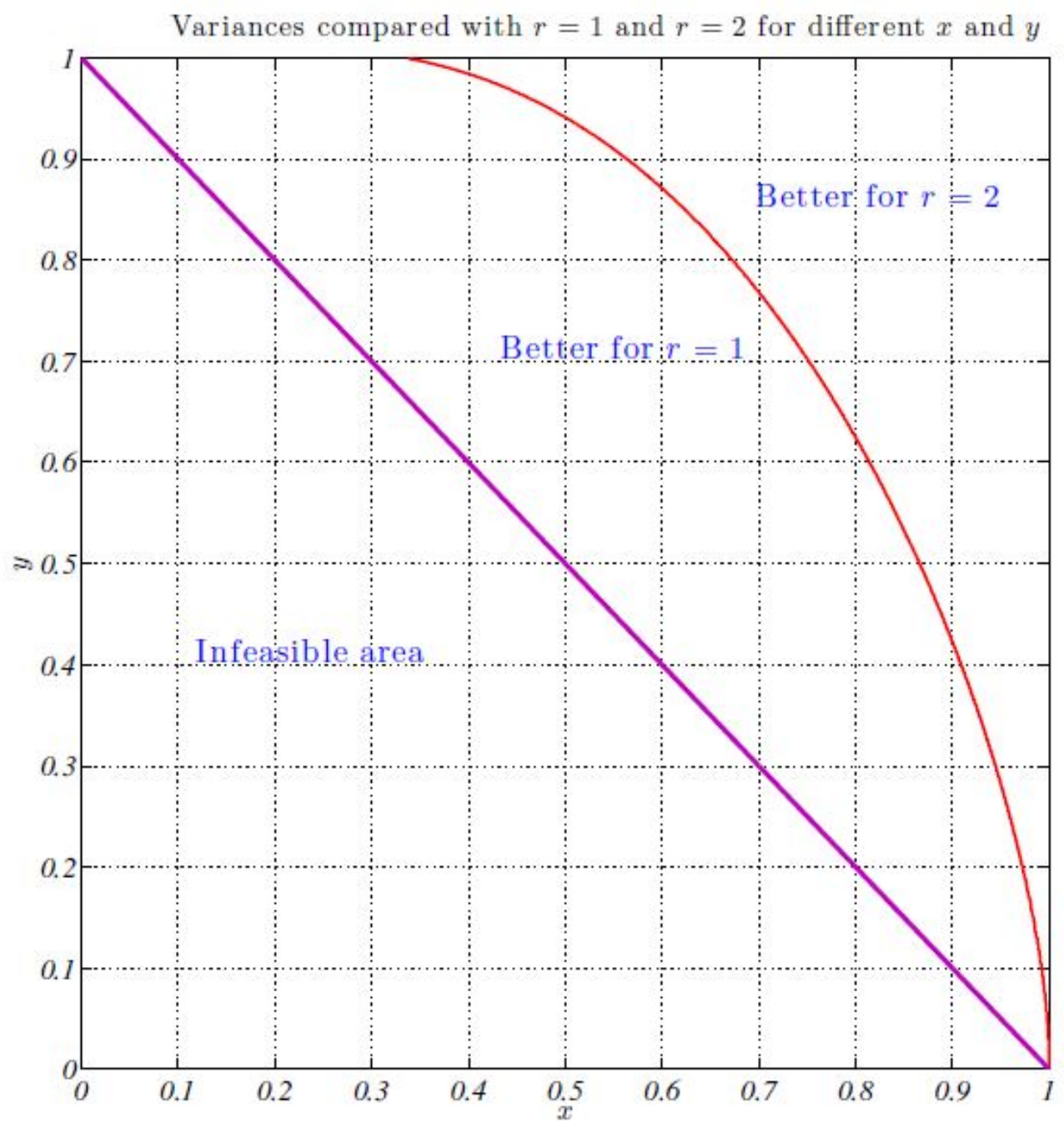


Figure 6: Optimal $r$ for different pairs of $x$ and $y$

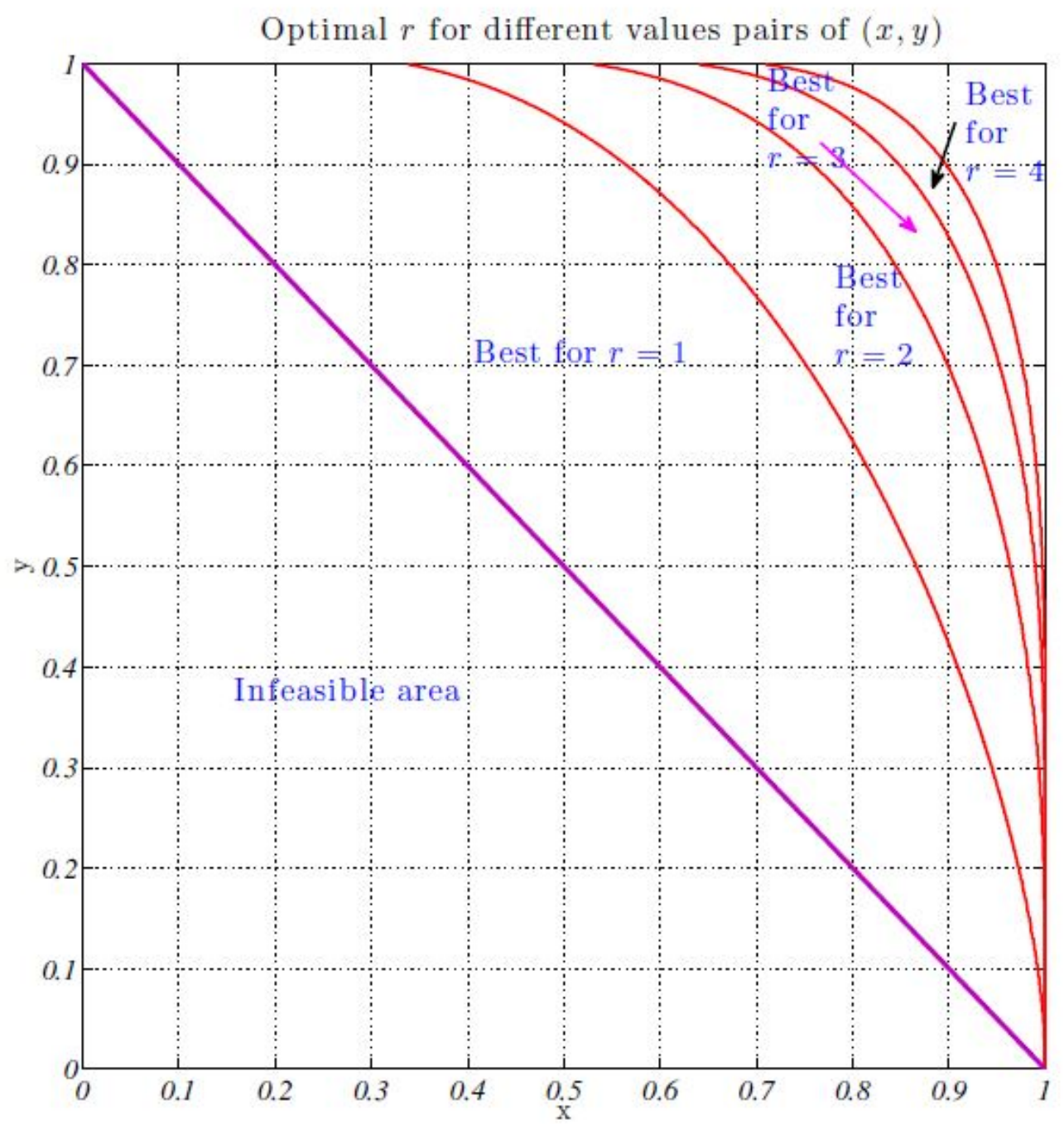


Figure 7: Histograms of the $\ell_{1}$ distances between the estimated $g$ and the true $g$ for nonaggregated data $D$ and time-aggregated data $D_{r}$

\section{Non-aggregated data}

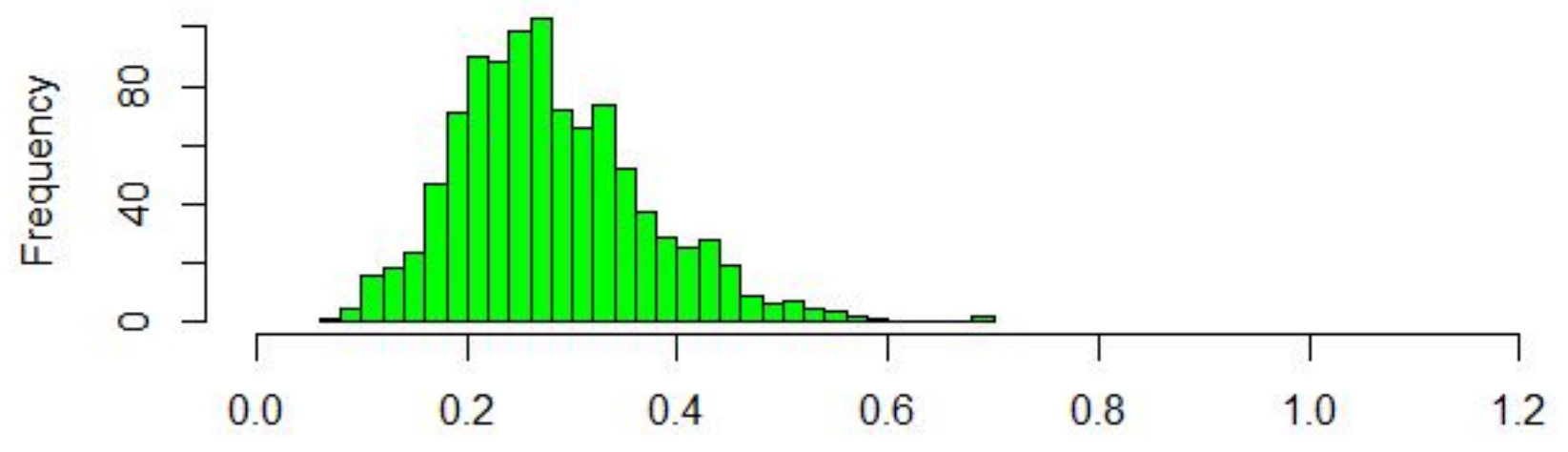

Time-aggregated data

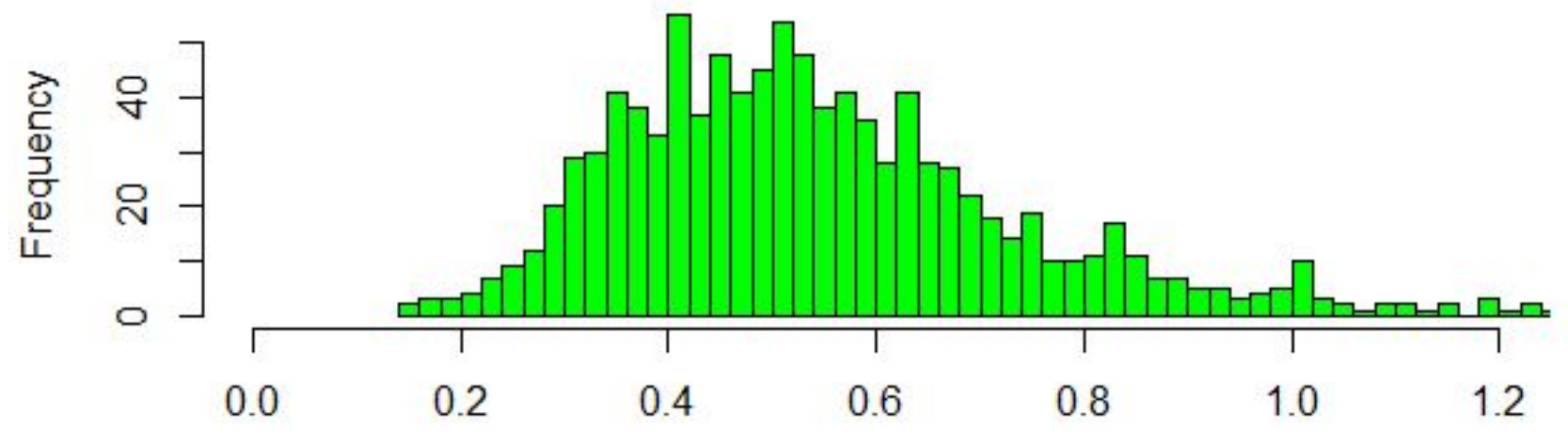


Figure 8: Histograms of the estimated replacement cost $\theta_{u}$ for four cases

Rust for non-aggregated data

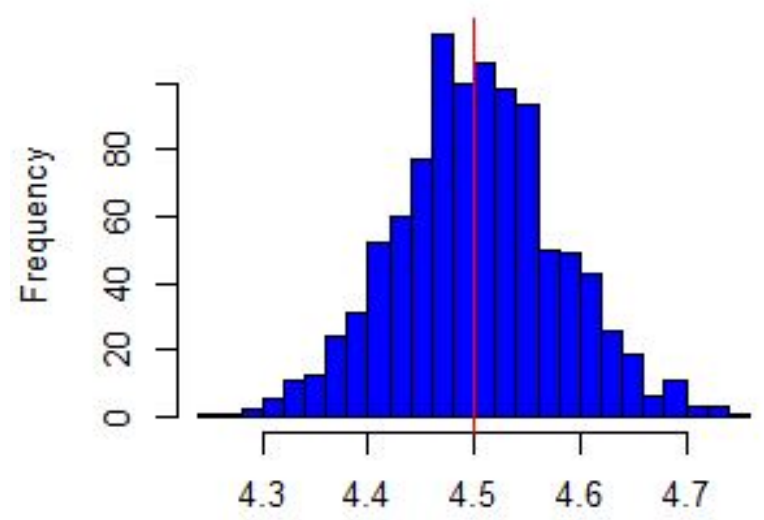

Hotz-Miller for non-aggregated data

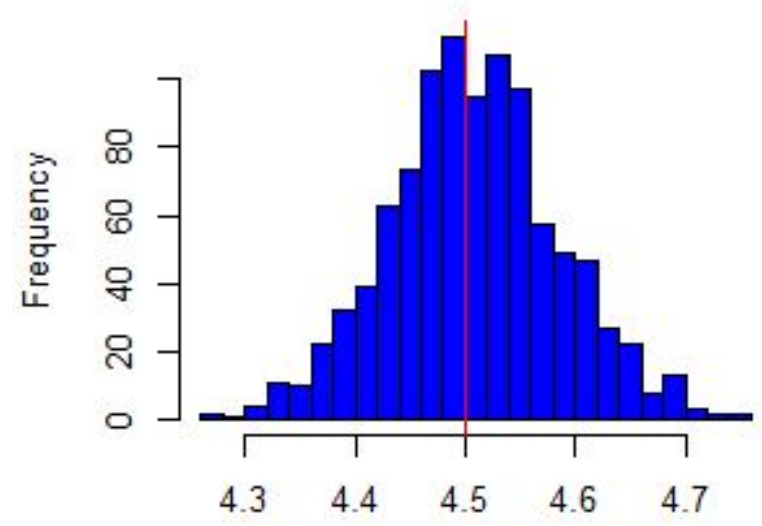

Rust for time-aggregated data

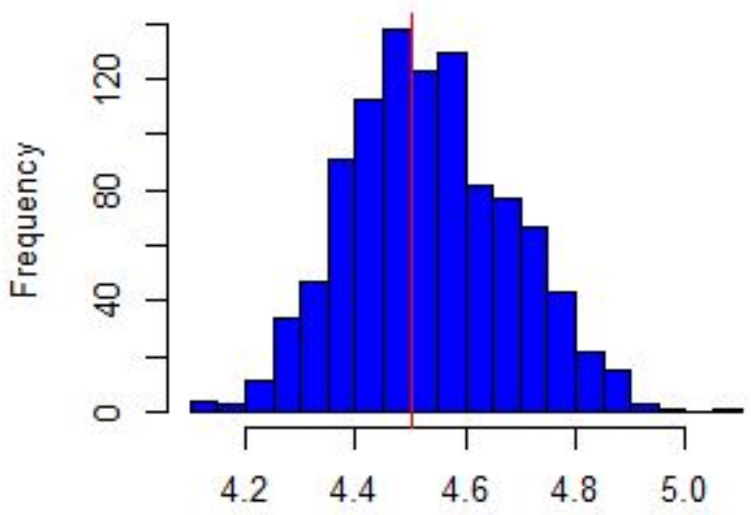

Hotz-Miller for time-aggregated data

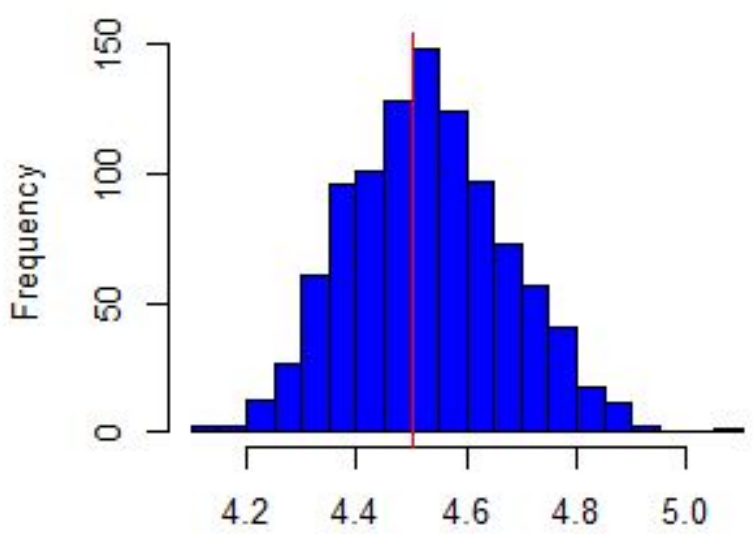

